\title{
Regularity for the One-Phase Hele-Shaw problem from a Lipschitz initial surface
}

\author{
Sunhi Choi, David Jerison and Inwon Kim
}

June 2, 2005

\begin{abstract}
In this paper we show that if the Lipschitz constant of the initial free boundary is small, then for small positive time the solution is smooth and satisfies the Hele-Shaw equation in the classical sense. A key ingredient in the proof which is of independent interest is an estimate up to order of magnitude of the speed of the free boundary in terms of initial data.
\end{abstract}

\section{Introduction}

Consider a compact set $K \subset \mathbb{R}^{n}$ with smooth boundary $\partial K$. Suppose that a bounded domain $\Omega$ contains $K$ and let $\Omega_{0}=\Omega-K$ and $\Gamma_{0}=\partial \Omega$ (Figure 1). Note that $\partial \Omega_{0}=\Gamma_{0} \cup \partial K$.

Let $u_{0}$ be the harmonic function in $\Omega_{0}$ with $u_{0}=f>0$ on $K$ and zero on $\Gamma_{0}$. Let $u(x, t)$ solve the one phase Hele-Shaw problem

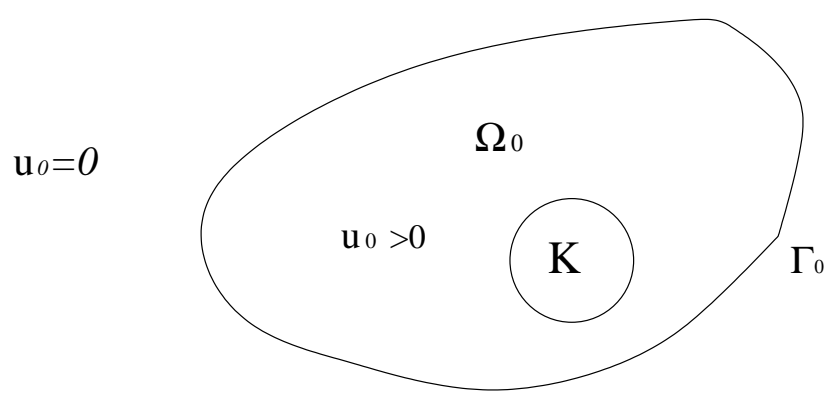

Figure 1. 


$$
\begin{cases}-\Delta u=0 & \text { in }\{u>0\} \cap Q, \\ u_{t}-|\nabla u|^{2}=0 & \text { on } \partial\{u>0\} \cap Q, \\ u(x, 0)=u_{0}(x) ; & u(x, t)=f \text { for } x \in \partial K .\end{cases}
$$

where $Q=\left(\mathbb{R}^{n}-K\right) \times(0, \infty)$. We refer to $\Gamma_{t}(u):=\partial\{u(\cdot, t)>0\}-\partial K$ as the free boundary of $u$ at time $t$ and to $\Omega_{t}(u):=\{u(\cdot, t)>0\}$ as the positive phase. Note that if $u$ is smooth up to the free boundary, then the free boundary moves with normal velocity $V=u_{t} /|\nabla u|$, and hence the second equation in (HS) implies that $V=|\nabla u|$. The classical Hele-Shaw problem models an incompressible viscous fluid which occupies part of the space between two parallel, nearby plates. The short-time existence of classical solutions when $\Gamma_{0}$ is $C^{2+\alpha}$ was proved by Escher and Simonett [ES]. When $n=2$, Elliot and Janovsky [EJ] showed the existence and uniqueness of weak solutions formulated by a parabolic variational inequality in $H^{1}(Q)$. For our investigation we use the notion of viscosity solutions introduced in [K1].(See also section 2.)

In this paper we investigate general Lipschitz domain $\Omega_{0}$ in $\mathbb{R}^{n}$ with Lipschitz constant less than a dimensional constant $a_{n}$. (In particular, $a_{2}=$ 1.) Our main result is that, for small time, $u$ is a classical solution of (HS) and the free boundary is smooth in space and time (Theorem 11.8). Suppose $0 \in \Gamma_{0}$ and define, for $P \in B_{1}(0) \cap\left(\mathbb{R}^{n}-\bar{\Omega}\right)$,

$$
t(P)=\sup \{t>0: u(P, t)=0\} .
$$

In other words $t(P)$ is the time the free boundary reaches $P$. Define $\delta=$ $\delta(P)=\operatorname{dist}(P, \bar{\Omega})$. Choose any point $z=z(P)$ in $\Omega$ such that $|P-z|=2 \delta$ and $\operatorname{dist}(z, \partial \Omega) \geq \delta / 2$. When $n=2$ and $\Gamma_{0}$ is a sector with (positive phase) angle between $\pi / 2$ and $2 \pi$, Jerison and Kim [JeKi] proved that

$$
t(P) \simeq \delta(P)^{2} / u_{0}(z(P)) .
$$

(Here $a \simeq b$ means that $a / b$ is bounded above and below by positive constants.) This result also easily extends to the case of radially symmetric cones in higher dimensions. The cone must have a sufficiently wide opening that the initial harmonic function $u_{0}$ tends to zero more slowly than $r^{2}$ at the vertex. (In dimension 2, this is the significance of the restriction to angles larger than the right angle.) In particular (0.1) implies that the average 
normal velocity of the free boundary moving from $P+z(P) / 2$ to $P$ between $t=0$ and $t=t(P)$ is comparable to

$$
u_{0}(z(P)) / \delta(P) \simeq\left|\nabla u_{0}(z(P))\right| .
$$

A key step in the proof, which is of independent interest, is the extension of (0.1) to Lipschitz initial domains, which yields an estimate up to order of magnitude of the speed of the free boundary in terms of initial condition. We show that $(0.1)$ holds at the infinitesimal level, that is, the normal velocity of the $\Gamma_{t}$ at $P$ at $t=t(P)$ is comparable to the average velocity $\mid \nabla u_{0}(z(P) \mid$ (Corollary 8.2 and Theorem 11.8).

Here is an outline of the paper. In section 1 some preliminary results are stated along with the definition and properties of viscosity solutions. A key tool is the comparison principle for viscosity solutions (Theorem 1.8). In section 2 we prove a Carleson-type estimate, which yields (0.1) for Lipschitz initial domains. We first prove the estimate for starshaped initial domains, and compare our solution with the ones on starshaped initial domains to obtain the general case. In fact, for starshaped, Lipschitz, initial domains we prove that the positive phase remains starshaped and Lipschitz in space for all time. This allows us to carry out all the estimates of subsequent sections of the paper in the special case of starshaped initial domains.

In section 3-5 we prove that the positive phase remains a Lipschitz domain in space for small time if the Lipschitz constant is sufficiently small. Following [C1] we show that $u$ is monotone in a cones of spatial directions which implies that all its level sets are Lipschitz graphs. The main idea is to prove first that at each time the level surfaces of $u$ are within $\epsilon$ of Lipschitz graphs (known as $\epsilon$-monotonicity of $u$ ) This is accomplished by comparison with solutions with starshaped initial domains. We then follow the argument in [C1] for improving the $\epsilon$-monotonicity to fully monotonicity of $u$. For this argument it is essential to have the nondegeneracy of $u$ on the free boundary at a scale corresponding to the $\epsilon$ above (see section 4). In section 6 we show that for $n=2$ a relatively simple reflection argument can be used to derive the monotonicity of $u$ in space for small time. When $n=2$ we only require the Lipschitz constant to be smaller than one. In section 7 , a lower bound of the speed on the free boundary is proven for positive small times. The rest of the paper is concerned with proving the upper bound on the speed of the free boundary. The non-uniformity of this bound makes this step challenging. Due to the free boundary motion law $V=|\nabla u|$, upper bounds on $u_{t}$ and $|\nabla u|$ are closely related. In section 8 we prove that $u_{t} \leq C|\nabla u|^{2}$, which yields an upper bound for the time derivative in the 
positive phase, away from the free boundary. In section 9 and 10 we apply an iteration process introduced by Caffarelli ([ACS],[C1]) to show that this interior upper bound propagates to the free boundary after a time delay. Moreover it is shown that the free boundary becomes smooth for positive small times and the solution satisfies (HS) in the classical sense.

\section{Preliminary results}

For a nonnegative real valued function $u(x, t)$ defined in a cylindrical domain $D \times(a, b)$, denote

$$
\begin{aligned}
& \Omega(u)=\{(x, t): u(x, t)>0\}, \quad \Omega_{t}(u)=\{x: u(x, t)>0\}, \\
& \Gamma(u)=\partial\{(x, t): u(x, t)=0\}, \quad \Gamma_{t}(u)=\partial\{x: u(x, t)=0\} .
\end{aligned}
$$

Let us recall the notion of viscosity solutions of (HS) defined in [K1]. Roughly speaking, viscosity sub and supersolutions are defined by comparison with local (smooth) super and subsolutions. In particular classical solutions of (HS) are also viscosity sub and supersolutions of (HS). Let $Q=\left(\mathbb{R}^{n}-K\right) \times(0, \infty)$ and let $\Sigma$ be a cylindrical domain $D \times(a, b) \subset \mathbb{R}^{n} \times \mathbb{R}$, where $D$ is an open subset of $\mathbb{R}^{n}$.

Definition 1.1. A nonnegative upper semicontinuous function u defined in $\Sigma$ is a viscosity subsolution of $(H S)$ if

(a) for each $a<T<b$ the set $\overline{\Omega(u)} \cap\{t \leq T\}$ is bounded; and

(b) for every $\phi \in C^{2,1}(\Sigma)$ such that $u-\phi$ has a local maximum in $\overline{\Omega(u)} \cap$ $\left\{t \leq t_{0}\right\} \cap \Sigma$ at $\left(x_{0}, t_{0}\right)$,

(i) $\quad-\Delta \phi\left(x_{0}, t_{0}\right) \leq 0 \quad$ if $u\left(x_{0}, t_{0}\right)>0$.

(ii) $\quad\left(\phi_{t}-|\nabla \phi|^{2}\right)\left(x_{0}, t_{0}\right) \leq 0 \quad$ if $\left(x_{0}, t_{0}\right) \in \Gamma(u)$ and $-\Delta \phi\left(x_{0}, t_{0}\right)>0$.

Note that because $u$ is only upper semicontinuous there may be points of $\Gamma(u)$ at which $u$ is positive.

Definition 1.2. A nonnegative lower semicontinuous function $v$ defined in $\Sigma$ is a viscosity supersolution of $(H S)$ if for every $\phi \in C^{2,1}(\Sigma)$ such that $v-\phi$ has a local minimum in $\Sigma \cap\left\{t \leq t_{0}\right\}$ at $\left(x_{0}, t_{0}\right)$, 
(i) $\quad-\Delta \phi\left(x_{0}, t_{0}\right) \geq 0 \quad$ if $v\left(x_{0}, t_{0}\right)>0$,

(ii) If $\left(z_{0}, t_{0}\right) \in \Gamma(v),|\nabla \phi|\left(x_{0}, t_{0}\right) \neq 0$ and $-\Delta \varphi\left(x_{0}, t_{0}\right)<0$,

then

$$
\left(\phi_{t}-|\nabla \phi|^{2}\right)\left(x_{0}, t_{0}\right) \geq 0 .
$$

Definition 1.3. $u$ is a viscosity subsolution of (HS) with initial data $u_{0}$ and fixed boundary data $f>0$ if

(a) $u$ is a viscosity subsolution in $Q$,

(b) $u$ is upper semicontinuous in $\bar{Q}, u=u_{0}$ at $t=0$ and $u \leq f$ on $\partial K$.

(c) $\overline{\Omega(u)} \cap\{t=0\}=\overline{\Omega\left(u_{0}\right)}$.

Definition 1.4. $u$ is a viscosity supersolution of $(H S)$ with initial data $u_{0}$ and fixed boundary data $f$ if $v$ is a viscosity supersolution in $Q$, lower semicontinuous in $\bar{Q}$ with $v=v_{0}$ at $t=0$ and $v \geq f$ on $\partial K$.

For a nonnegative real valued function $u(x, t)$ defined in a cylindrical domain $D \times(a, b)$,

$$
u^{*}(x, t)=\limsup _{(\xi, s) \in D \times(a, b) \rightarrow(x, t)} u(\xi, s) .
$$

Note that the limsup permits times in the future of $t, s>t$.

Definition 1.5. $u$ is a viscosity solution of (HS) (with boundary data $u_{0}$ and $f$ ) if $u$ is a viscosity supersolution and $u^{*}$ is a viscosity subsolution of (HS) (with boundary data $u_{0}$ and $f$.)

Definition 1.6. We say that a pair of functions $u_{0}, v_{0}: \bar{D} \rightarrow[0, \infty)$ are (strictly) separated (denoted by $u_{0} \prec v_{0}$ ) in $D \subset \mathbb{R}^{n}$ if

(i) the support of $u_{0}, \operatorname{supp}\left(u_{0}\right)=\overline{\left\{u_{0}>0\right\}}$ restricted in $\bar{D}$ is compact and

(ii) in $\operatorname{supp}\left(u_{0}\right) \cap \bar{D}$ the functions are strictly ordered:

$$
u_{0}(x)<v_{0}(x)
$$


Definition 1.7. $\Omega$ is a Lipschitz domain with Lipschitz constant $\bar{M}$ if there are constants $0<\bar{r}, \bar{R}<\infty$ such that the diameter of $\Omega$ is less than $\bar{R}$ and for any $x_{0} \in \partial \Omega$ we have, after rotation and translation,

$$
\Omega \cap B_{\bar{r}}\left(x_{0}\right)=\left\{\left(x^{\prime}, x_{n}\right) \in \mathbb{R}^{n-1} \times \mathbb{R}: x_{n}>f\left(x^{\prime}\right)\right\} \cap B_{\bar{r}}\left(x_{0}\right) .
$$

where $f$ is Lipschitz with Lipschitz constant less than $\bar{M}$.

The following properties of viscosity solutions are frequently used in our paper.

Theorem 1.8. (Comparison principle, [K2]) Let $u, v$ be respectively viscosity sub- and supersolutions in $D \times(0, T) \subset Q$ with initial data $u_{0} \prec v_{0}$ in $D$. If $u \leq v$ on $\partial D$ and $u<v$ on $\partial D \cap \bar{\Omega}(u)$ for $0 \leq t<T$, then $u(\cdot, t) \prec v(\cdot, t)$ in $D$ for $t \in[0, T)$.

For $x \in \mathbb{R}^{n}, B_{r}(x):=\left\{y \in \mathbb{R}^{n}:|y-x|<r\right\}$. For simplicity we will consider the case $f=1$ and $K=B_{r}(0)$ for some $r>0$.

Theorem 1.9. (a) For $\Omega_{0}$ with small Lipschitz constant $\bar{M}$, there is a unique viscosity solution $u$ in $Q$ with boundary data 1 and initial data $u_{0}$.

(b) $u$ is harmonic in $\Omega(u)$. Indeed $u(x, t)=h_{t}(x)$, where

$$
h_{t}(x)=\inf \left\{v \in \mathcal{P} \text { with } v=1 \text { on } \partial K \text { and } v \geq 0 \text { on } \Gamma_{t}\right\} \text {. }
$$

where $\mathcal{P}$ is the set of superharmonic functions in $\Omega_{t}$ which are lowersemicontinuous in $\bar{\Omega}_{t}$.

Proof. Part (a) follows from Theorem 1.8, the proof of Theorem 3.3 of [K1] and Lemma 3.6 of [JeKi].

Now to prove (b), let $h(x, t)=h_{t}(x)$ be defined as above for $t \geq 0$. Then it follows that $h(\cdot, t)$ is harmonic in $\Omega_{t}(u)$ (see Chapter 1.3 of $[\mathrm{T}]$ for example.) Since $u$ is superharmonic in $\Omega(u)$ by definition, From definition of $h$ it follows that $h(x, t) \leq u(x, t)$. On the other hand by Theorem 1.8 $u^{*}(x, t-\epsilon) \leq u(x, t)$ for $t>\epsilon$, and thus $u^{*}(x, t-\epsilon)=0$ on $\Gamma_{t}(u)$. Thus again by definition of $h$ we obtain $u^{*}(x, t-\epsilon) \leq h(x, t)$ for any small $\epsilon>0$. Now it follows from the lower semicontinuity of $u$ that

$$
u(x, t) \leq \lim _{\epsilon \rightarrow 0} u^{*}(x, t-\epsilon) \leq h(x, t) \quad \text { for } t>0,
$$

which leads to $h=u$ for $t \geq 0$. 
Next we state several properties of harmonic functions.

Lemma 1.10. (Dahlberg, $[D])$ Let $u_{1}, u_{2}$ be two nonnegative harmonic functions in a domain $D$ of $\mathbb{R}^{n}$ of the form

$$
D=\left\{\left(x^{\prime}, x_{n}\right) \in \mathbb{R}^{n-1} \times \mathbb{R}:\left|x^{\prime}\right|<2,\left|x_{n}\right|<2 \bar{M}, x_{n}>f\left(x^{\prime}\right)\right\}
$$

with $f$ a Lipschitz function with constant less than $\bar{M}$ and $f(0)=0$. Assume further that $u_{1}=u_{2}=0$ along the graph of $f$. Then for

$$
D_{1 / 2}=\left\{\left|x^{\prime}\right|<1,\left|x_{n}\right|<\bar{M}, x_{n}>f\left(x^{\prime}\right)\right\}
$$

We have

$$
0<C_{1} \leq \frac{u_{1}\left(x^{\prime}, x_{n}\right)}{u_{2}\left(x^{\prime}, x_{n}\right)} \cdot \frac{u_{2}(0, \bar{M})}{u_{1}(0, \bar{M})} \leq C_{2}
$$

with $C_{1}, C_{2}$ depending only on $\bar{M}$.

Lemma 1.11. (Caffarelli, [C1]) Let $u$ be as in Lemma 1.10. Then there exists $c, C_{1}, C_{2}>0$ depending only on $\bar{M}$ such that for $0<d<c \frac{\partial}{\partial x_{n}} u(0, d) \geq$ 0 and

$$
C_{1} \frac{u(0, d)}{d} \leq \frac{\partial u}{\partial x_{n}}(0, d) \leq C_{2} \frac{u(0, d)}{d}
$$

Lemma 1.12. (Caffarelli, [C1]) Let u be harmonic in $B_{1}$. Then there exists $\epsilon_{0}>0$ such that if

$$
u(x+\epsilon e) \geq u(x) \text { for } \epsilon>\epsilon_{0} \text { and } x, x+\epsilon e \in B_{1}(0)
$$

for a unit vector $e \in \mathbb{R}^{n}$ then $e \cdot \nabla u \geq 0$ in $B_{1 / 2}(0)$.

Lemma 1.13. ([JK], Lemma 4.1) Let $\Omega$ be Lipschitz domain contained in $B_{10}(0)$. There exists a dimensional constant $\beta_{n}>0$ such that for any $\zeta \in \partial \Omega, 0<2 r<1$ and positive harmonic function $u$ in $\Omega \cap B_{2 r}(\zeta)$, if $u$ vanishes continuously on $B_{2 r}(\zeta) \cap \partial \Omega$, then for $x \in \Omega \cap B_{r}(\zeta)$,

$$
u(x) \leq C\left(\frac{|x-\zeta|}{r}\right)^{\beta_{n}} \sup \left\{u(y): y \in \partial B_{2 r}(\zeta) \cap \Omega\right\}
$$

where $C$ depends only on the Lipschitz constants of $\Omega$. 


\section{A Carleson-type estimate}

In this section we prove a Carleson type estimate for (HS), that is, if $u(x, t)$ is a solution of (HS) with $\Omega$ being a Lipschitz domain with a small Lipschitz constant, then $u_{0}$ evaluated at $P \in \Omega$ controls $\sup \left\{u(x, t): x \in B, t \leq t_{0}\right\}$ where $B$ is a box with the center on $\Gamma_{0}$ and containing $P$ in the middle of $B \cap \Omega_{0}$ and $t_{0}$ is the time when the free boundary of $u$ escapes $B$.

For $x=\left(x^{\prime}, x_{n}\right) \in \Gamma_{0}$, we let

$$
H^{u}(x, t)=\inf \left\{d: y=\left(x^{\prime}, x_{n}+d\right) \in \Gamma_{t}(u)\right\}
$$

where the coordinate $\left(x^{\prime}, x_{n}\right)$ is given so that near $x$

$$
\Gamma_{0}=\left\{\left(x^{\prime}, x_{n}\right): x^{\prime} \in \mathbb{R}^{n-1}, x_{n}=f\left(x^{\prime}\right)\right\} .
$$

When the reference to the function is clear, we will denote $H(x, t)=H^{u}(x, t)$.

For $P \in \mathbb{R}^{n}-\Omega$,

$$
t(P, u)=\sup \{t>0: u(P, t)=0\} .
$$

When the reference to the function is clear we will denote $t(P):=t(P, u)$.

We say $a$ is comparable to $b$ or $a \approx b$, if $\frac{1}{C} a \leq b \leq C a$ with a dimensional constant $C$.

We say $a$ is comparable to $b$ depending on the global properties of the initial Lipschitz domain and write

$$
a \stackrel{g}{\approx} b
$$

if $\frac{1}{C} a \leq b \leq C a$ for a positive constant $C$ only depending on the dimension $n$ and the constants $\bar{r}, \bar{R}, \bar{M}$ associated with the initial Lipschitz domain $\Omega$.

For a unit vector $\nu \in \mathbb{R}^{n}$ and $0<\theta \leq \pi / 2$, denote the cone with axis $\nu$ and central angle $\theta$ by

$$
W(\theta, \nu):=\left\{x \in \mathbb{R}^{n}:(x, \nu) \geq|x| \cos \theta\right\} .
$$

For $n \geq 3$ define a dimensional constant $a_{n}>0$ such that if $h(x)$ is a nonnegative harmonic function in $W\left(\theta,-e_{n}\right)$ with $\pi / 2-\theta<2 a_{n}$ and $h=0$ on the boundary of $W\left(\theta,-e_{n}\right)$, then the maximal and the minimal decay rate of $h$ at 0 is between $5 / 6$ and $7 / 6$, i.e.,

$$
d^{7 / 6} \leq h\left(-d e_{n}\right) \leq d^{5 / 6}
$$

if $d>0$ is sufficiently small. For $n=2$, define $a_{2}=1$. 
Theorem 2.1. Suppose $u$ solves (HS) with the initial domain $\Omega_{0}=\Omega-K \subset$ $\mathbb{R}^{n}$ where $\Omega$ is a Lipschitz domain with constants $\bar{r}, \bar{R}$ and $\bar{M}$ such that $\bar{M}<a_{n}$. Let $P_{0} \in \Gamma_{0}$ and $0<\delta_{0}<h \bar{r}$ where $h>0$ is a constant depending only on $\bar{r}, \bar{R}$ and the dimension $n$. Let

$$
T=\sup \left\{t: \Gamma_{t} \cap B_{\delta_{0}}\left(P_{0}\right) \neq \emptyset\right\}
$$

then for any $P_{1} \in \Omega_{0}$ such that $\left|P_{1}-P_{0}\right| \approx \operatorname{dist}\left(P_{1}, \Gamma_{0}\right) \approx \delta_{0}$,

$$
\sup _{x \in B_{\delta_{0}}\left(P_{0}\right)} u(x, T) \leq C u\left(P_{1}, 0\right)
$$

for $C$ depending only on $\bar{r}, \bar{R}, \bar{M}$ and the dimension n. (see Figure 2.)

Corollary 2.2. Under the same assumption as in Theorem 2.1, let $P_{1} \in \Omega_{0}$ and $P_{2} \in \Omega_{0}^{c}$ satisfy

$$
\delta_{0} \approx\left|P_{1}-P_{0}\right| \approx \operatorname{dist}\left(P_{1}, \Gamma_{0}\right) \approx\left|P_{2}-P_{0}\right| \approx \operatorname{dist}\left(P_{2}, \Gamma_{0}\right) .
$$

Then

$$
t\left(P_{2}\right) \stackrel{g}{\approx} \frac{\delta_{0}^{2}}{u\left(P_{1}, 0\right)}
$$

Remark. By Lemma 1.11,

$$
\frac{u\left(P_{1}, 0\right)}{\delta_{0}} \stackrel{g}{\approx}\left|\nabla u\left(P_{1}, 0\right)\right|
$$

Roughly speaking, Corollary 2.2 says that the average speed of $\Gamma$ travelling from $P_{0}$ to $P_{2}$ is comparable to $\left|\nabla u\left(P_{1}, 0\right)\right|$, which is, in turn, an average of $|\nabla u|$ in a $\delta$-neighborhood of $P_{0}$. Indeed the average speed of $\Gamma$ is $\delta_{0} / t\left(P_{2}\right)$, and Lemma 1.11 implies $u\left(P_{1}, 0\right) / \delta_{0} \simeq\left|\nabla u\left(P_{1}, 0\right)\right|$. Hence, substituting into Corollary 2.2 gives

$$
\frac{\delta_{0}}{t\left(P_{2}\right)} \stackrel{g}{\approx}\left|\nabla u\left(P_{1}, 0\right)\right|
$$

Corollary 2.3. Under the same assumption as in Theorem 2.1, $u(x, t)$ is Hölder continuous in time. More precisely, for $x \in \Omega_{0}$ and $P_{x}=x+r_{x} e_{n} \in$ $\Gamma_{0}\left(0<r_{x}<\bar{r}\right)$ if $0 \leq s \leq r_{x}$ then

$$
\frac{u\left(x, t\left(P_{x}+s e_{n}\right)\right)}{u(x, 0)} \leq 1+C\left(\frac{s}{r_{x}}\right)^{\beta_{n}}
$$

where $0<\beta_{n}<1$ is a dimensional constant depending on $\bar{M}$ and $C>0$ is a constant depending only on $\bar{r}, \bar{R}, \bar{M}$ and dimension $n$. 
Proof of Theorem 2.1 We divide into two cases. First we prove the theorem when $\Omega$ is star-shaped, and then proceed to the general case.

Case 1 Let $\Omega$ be a star-shaped region with respect to every $x \in K$ for a sufficiently large ball $K \subset \Omega$. First, we show that the flow preserves starshapedness. This lemma will be also used in section 3 .

Lemma 2.4. Let $\Omega$ contain $K=B_{1}(0)$ and star-shaped with respect to $x_{0} \in K$. Let $v(x, t)$ be the viscosity solution of (HS) with initial domain $\Omega-K$ and fixed boundary data 1 on $\partial K$. Then $\Omega_{t}(v)$ is star-shaped with respect to $x_{0}$ for $t>0$

Proof. For given $\epsilon>0$, let us define

$$
\tilde{v}(x, t):=(1+\epsilon) v\left((1+\epsilon)^{-1}\left(x-x_{0}\right)+x_{0},(1+\epsilon) t\right) .
$$

Note that $\tilde{v}$ is a supersolution of (HS) in

$$
Q^{\epsilon}:=\left(\mathbb{R}^{n}-K^{\epsilon}\right) \times[0, \infty), K^{\epsilon}:=\left\{x \in \mathbb{R}^{n}:(1+\epsilon)^{-1}\left(x-x_{0}\right)+x_{0} \in K\right\} .
$$

We will apply the comparison principle (Theorem 1.8) for $v^{*}$ and $\tilde{v}$ in $Q^{\epsilon}$. First observe that on $\partial K^{\epsilon}$ we have

$$
v^{*}(x, t) \leq 1<\tilde{v}(x, t) .
$$

Secondly at $t=0$ we have $v^{*} \prec \tilde{v}$ by our hypothesis and the maximum principle for harmonic functions. Therefore by the comparison principle (Theorem 1.8), $v^{*} \leq \tilde{v}$ in $\left(\mathbb{R}^{n}-K^{\epsilon}\right) \times[0, \infty)$. It follows that $(1+\epsilon)^{-1}(x-$ $\left.x_{0}\right)+x_{0} \in \Omega_{t}(v)$ for every $x \in \Omega_{t}(v)$, and hence $\Omega_{t}(v)$ is star-shaped with respect to $x_{0}$ for $t>0$.

Remark If a domain is star-shaped with respect to every point in a ball inside the domain, it follows that the domain is Lipschitz. In particular Lemma 2.4 implies that $\Omega_{t}(v)$ is Lipschitz for every $t>0$.

Note that the $s=H^{u}\left(P_{0}, t\left(P_{0}+s e_{n}\right)\right)$ is the distance travelled by $P_{0}$ in time $t\left(P_{0}+s e_{n}\right)$. In Lemma 2.5, we find an upper bound for $H^{u}\left(Q_{0}, t\left(P_{0}+\right.\right.$ $\left.s e_{n}\right)$ ), i.e., the distance travelled by $Q_{0}$ in time $t\left(P_{0}+s e_{n}\right)$, in terms of $s$ and $\operatorname{dist}\left(P_{0}, Q_{0}\right)$ for any $Q_{0} \in \Gamma_{0} \cap B_{\bar{r}}\left(P_{0}\right)-B_{s}\left(P_{0}\right)$. In Lemma 2.6, we compare $u_{0}\left(P_{1}\right)$ and $u\left(P_{1}, t\left(P_{0}+s e_{n}\right)\right)$ using the distance between their boundaries. Observe that, by Theorem $1.9, u(\cdot, t)$ is harmonic in $\Omega_{t}$ with boundary data 0 on $\Gamma_{t}$ and 1 on $\partial K$. 


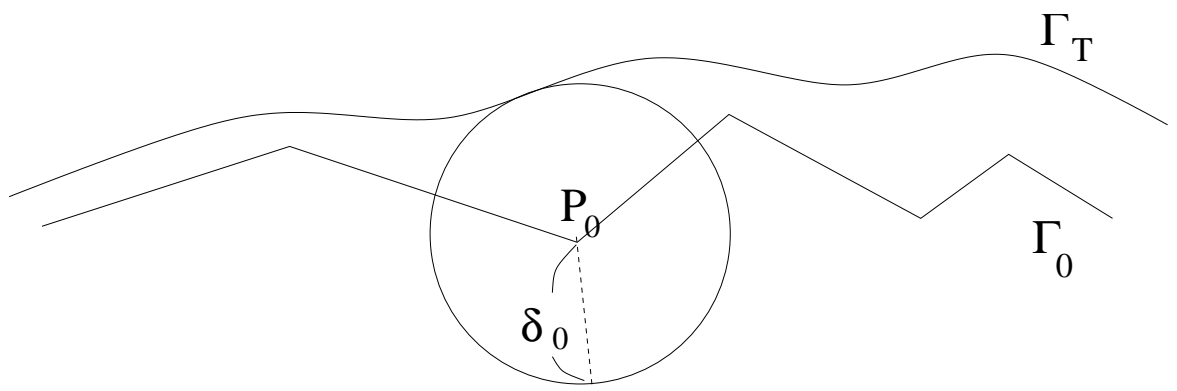

Figure 2.

Lemma 2.5. There exist a dimensional constant $C>0, h=h(\bar{r}, \bar{R}, n)>0$ and $\alpha=\alpha(\bar{M}, n)<1$ such that for any $P_{0} \in \Gamma_{0}$ and $Q_{0} \in \Gamma_{0} \cap B_{\bar{r}}\left(P_{0}\right)-$ $B_{s}\left(P_{0}\right), 0<s<h \bar{r}$,

$$
\frac{H^{u}\left(Q_{0}, t\left(P_{0}+s e_{n}\right)\right)}{s} \leq C\left(\frac{\operatorname{dist}\left(P_{0}, Q_{0}\right)}{s}\right)^{\alpha} .
$$

(see figure 3.)

Proof. We prove the lemma in Case 1, i.e., when $\Omega$ is a star-shaped region. If $\Omega$ is star-shaped, then by Lemma $2.4, \Gamma_{t}$ remains Lipschitz.

Denote $l=\operatorname{dist}\left(P_{0}, Q_{0}\right)$. Let $T^{P_{0}}$ be the cone with vertex $P_{0}$ and central angle $\arctan \frac{1}{\bar{M}}$ such that $T^{P_{0}} \cap B_{l}\left(P_{0}\right) \subset \Omega_{0}$. (see figure 3.) Also let $B^{P_{0}}$ be a ball in $T^{P_{0}} \cap B_{l}\left(P_{0}\right)$ such that

$$
l / 5<\operatorname{rad}\left(B^{P_{0}}\right) \approx \operatorname{dist}\left(B^{P_{0}}, \partial\left(T^{P_{0}} \cap B_{l}\left(P_{0}\right)\right)\right)<l .
$$

Let $B^{Q_{0}}=B_{l}\left(Q_{0}\right)$ and let $T^{Q_{0}}$ be the cone with vertex $Q_{0}$ and central angle $\arctan \frac{1}{\bar{M}}$ such that $T^{Q_{0}} \cap B_{l / 2}\left(Q_{0}\right) \subset \Omega_{0}^{c}$.

Let $v$ be the solution to (HS) with initial domain $T^{P_{0}} \cap B_{l}\left(P_{0}\right)-B^{P_{0}}$ and fixed boundary data $a_{0}$ on $\partial B^{P_{0}}$ where

$$
a_{0}=\inf \left\{u(x, 0): x \in \partial B^{P_{0}}\right\} .
$$

Also let $w$ be the solution to (HS) with initial domain $B^{Q_{0}}-T^{Q_{0}} \cap B_{l / 2}\left(Q_{0}\right)$ and fixed boundary data $C_{1}^{2} C_{2} a_{0}$ on $\partial B^{Q_{0}}$ where $C_{1}$ and $C_{2}$ are dimensional constants which will be determined later. We will use the time scaling $A v(x, A t)$ to adjust the size of $v$ to be less than, but comparable to $u$ on 

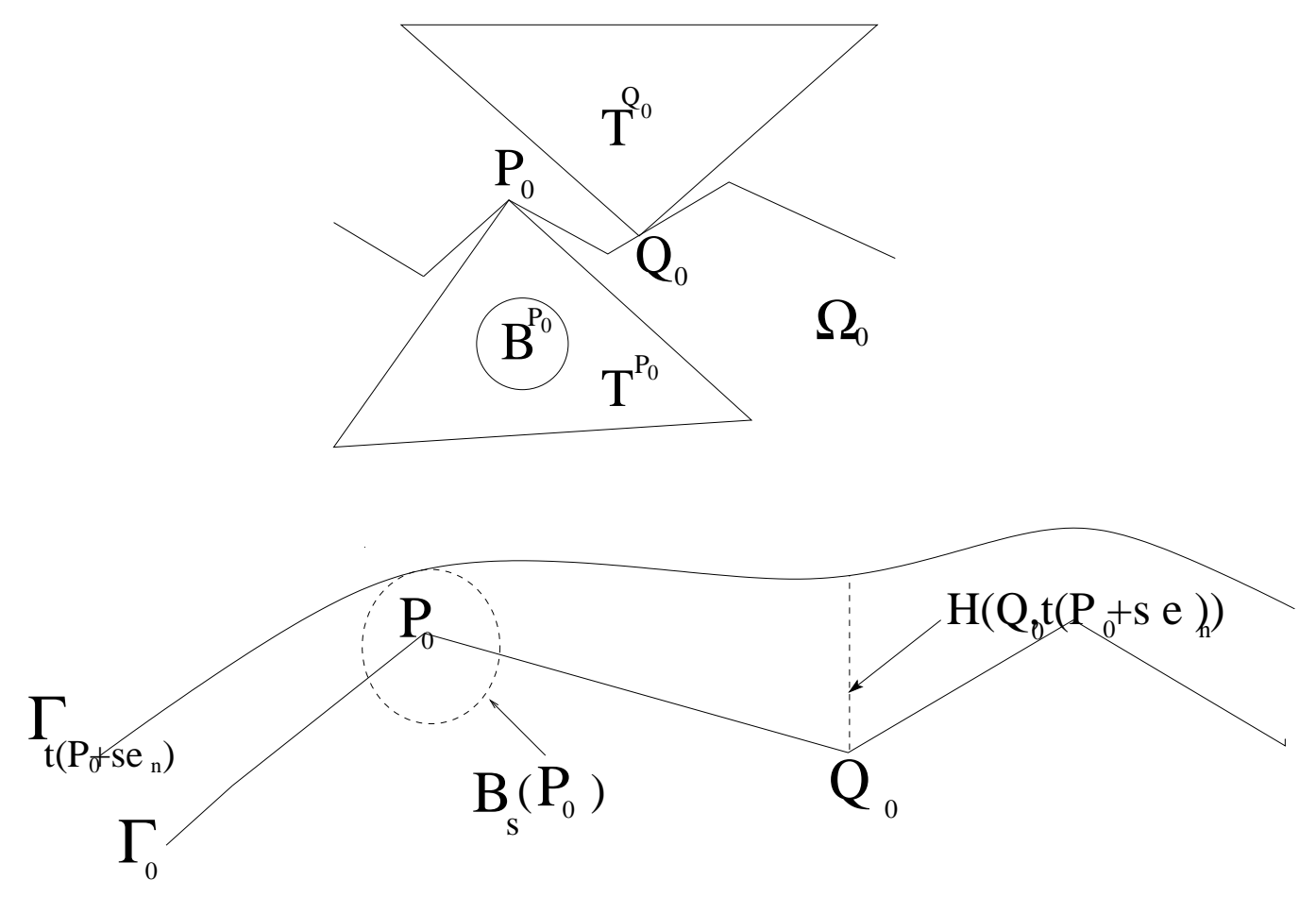

Figure 3. 
$\partial B^{P_{0}}$ to get a lower bound for the distance traveled by $P_{0}$. Similarly, a time scaled version of $w$ will give an upper bound for the distance traveled by $Q_{0}$.

By Harnack inequality, there is a dimensional constant $C_{1}$ such that

$$
\sup _{x \in B^{P_{0}}} u(x, t) \leq C_{1} \inf _{x \in B^{P_{0}}} u(x, t) .
$$

Furthermore $u(x, t)$ is increasing in $t$. Therefore, one can choose a sequence $0=t_{0}<t_{1}<\ldots<t_{k}=t\left(P_{0}+s e_{n}\right)$ such that for every $x \in \partial B^{P_{0}}$ and $t \in\left[t_{i-1}, t_{i}\right], 1 \leq i \leq k$

$$
C_{1}^{i-1} a_{0} \leq u(x, t) \leq C_{1}^{i+1} a_{0} .
$$

We will define $\left\{P_{i}\right\}_{i=1}^{k}$ inductively by changing the initial free boundary and the data on the fixed boundary at each step. First, replace $\Gamma_{0}$ with $\partial\left(T^{P_{0}} \cap B_{l}\left(P_{0}\right)\right)$ and $K$ with $B^{P_{0}}$, i.e., let $T^{P_{0}} \cap B_{l}\left(P_{0}\right)-B^{P_{0}}$ be the new domain. Denote $\partial\left(T^{P_{0}} \cap B_{l}\left(P_{0}\right)\right)$ by $\Gamma_{0}^{\prime}$ and let $\Gamma_{i}^{\prime}(1 \leq i \leq k)$ be the free boundary which has evolved from $\Gamma_{i-1}^{\prime}$ on the time interval $\left[0, t_{i}-t_{i-1}\right]$ with fixed boundary data $C_{1}^{i-1} a_{0}$ on $\partial B^{P_{0}}$. For $1 \leq i \leq k$, define

$$
r_{i}:=\inf \left\{r>0: P_{i-1}+r e_{n} \in \Gamma_{i}^{\prime}\right\}
$$

and

$$
P_{i}:=P_{i-1}+r_{i} e_{n} .
$$

Then since the new domain $T^{P_{0}} \cap B_{l}\left(P_{0}\right)-B^{P_{0}}$ is contained in $\Omega_{0}$ and the fixed boundary data $C_{1}^{i-1} a_{0} \leq \inf \left\{u(x, t):(x, t) \in \partial B^{P_{0}} \times\left[t_{i-1}, t_{i}\right]\right\}$, by the comparison principle (Theorem 1.8), $\Gamma_{k}^{\prime} \subset \Omega_{t_{k}}(u)$. In particular $P_{k} \in \Omega_{t_{k}}(u)$ and

$$
\operatorname{dist}\left(P_{0}, P_{k}\right) \leq H^{u}\left(P_{0}, t_{k}\right)=s .
$$

At each step we are just multiplying the fixed boundary data on $\partial B^{P_{0}}$ by $C_{1}$. Hence it follows from the scaling property of (HS) that

$$
\begin{gathered}
\operatorname{dist}\left(P_{0}, P_{k}\right)=H^{v}\left(P_{0}, t_{k}^{\prime}\right) \\
t_{k}^{\prime}=\sum_{i=1}^{k} C_{1}^{i}\left(t_{i}-t_{i-1}\right) .
\end{gathered}
$$

Recall that $v(x, t)$ is the solution to (HS) with initial domain $T^{P_{0}} \cap B_{l}\left(P_{0}\right)-$ $B^{P_{0}}$ and fixed boundary data $a_{0}$ on $\partial B^{P_{0}}$. 
On the other hand, since $\Gamma_{t}$ stays Lipschitz in $B^{Q_{0}}$, by Carleson Lemma and Harnack inequality, there exists a dimensional constant $C_{2}$ such that

$$
\sup \left\{u(x, t): x \in B^{Q_{0}}\right\} \leq C_{2} \inf \left\{u(x, t): x \in B^{P_{0}}\right\} .
$$

We define $\left\{Q_{i}\right\}$ similarly to $\left\{P_{i}\right\}$ by replacing the new initial domain with $B^{Q_{0}}-T^{Q_{0}} \cap B_{l / 2}\left(Q_{0}\right)$ and giving fixed boundary data $C_{1}^{i+1} C_{2} a_{0}$ on $\partial B^{Q_{0}}$ at each step.

Then by (2.2) and (2.5), for $t \in\left[t_{i-1}, t_{i}\right](1 \leq i \leq k)$,

$$
\sup \left\{u(x, t): x \in B^{Q_{0}}\right\} \leq C_{1}^{i+1} C_{2} a_{0} .
$$

Hence by the comparison principle (Theorem 1.8),

$$
H^{u}\left(Q_{0}, t_{k}\right) \leq \operatorname{dist}\left(Q_{0}, Q_{k}\right) .
$$

By the same reasoning as in (2.4)

$$
\operatorname{dist}\left(Q_{0}, Q_{k}\right)=H^{w}\left(Q_{0}, t_{k}^{\prime}\right)
$$

for the same $t_{k}^{\prime}$ as in (2.4) and $w(x, t)$ solving (HS) with initial domain $B^{Q_{0}}-T^{Q_{0}} \cap B_{l / 2}\left(Q_{0}\right)$ and fixed boundary data $C_{1}^{2} C_{2} a_{0}$ on $\partial B^{Q_{0}}$.

Let $t_{k}^{\prime \prime}$ be the time satisfying

$$
H^{v}\left(P_{0}, t_{k}^{\prime \prime}\right)=s .
$$

Then we obtain $t_{k}^{\prime \prime} \geq t_{k}^{\prime}$ since $(2.3),(2.4)$ and $t_{k}=t\left(P_{0}+s e_{n}\right)$ imply that

$$
H^{v}\left(P_{0}, t_{k}^{\prime}\right) \leq H^{u}\left(P_{0}, t_{k}\right)=H^{u}\left(P_{0}, t\left(P_{0}+s e_{n}\right)\right)=s .
$$

Also by (2.6) and (2.7),

$$
\frac{H^{u}\left(Q_{0}, t_{k}\right)}{s} \leq \frac{H^{w}\left(Q_{0}, t_{k}^{\prime}\right)}{s} \leq \frac{H^{w}\left(Q_{0}, t_{k}^{\prime \prime}\right)}{s}=\frac{H^{w}\left(Q_{0}, t_{k}^{\prime \prime}\right)}{H^{v}\left(P_{0}, t_{k}^{\prime \prime}\right)}
$$

where the second inequality comes from the fact that $t_{k}^{\prime \prime} \geq t_{k}^{\prime}$. By a scaled version of results on cones in [JeKi], there exist $\beta_{1}=\beta_{1}(\bar{M}, n)<1$ and $\beta_{2}=\beta_{2}(\bar{M}, n)>1$ such that

$$
H^{w}\left(Q_{0}, t_{k}^{\prime \prime}\right) \approx l\left(\frac{C_{1} C_{2} a_{0} t_{k}^{\prime \prime}}{l^{2}}\right)^{\beta_{1}}
$$


and

$$
H^{v}\left(P_{0}, t_{k}^{\prime \prime}\right) \approx l\left(\frac{a_{0} t_{k}^{\prime \prime}}{l^{2}}\right)^{\beta_{2}} .
$$

Since $s=H^{v}\left(P_{0}, t_{k}^{\prime \prime}\right)$, the right side of (2.8) satisfies the following:

$$
\begin{aligned}
\frac{H^{w}\left(Q_{0}, t_{k}^{\prime \prime}\right)}{H^{v}\left(P_{0}, t_{k}^{\prime \prime}\right)} & \approx\left(\frac{a_{0} t_{k}^{\prime \prime}}{l^{2}}\right)^{\beta_{1}-\beta_{2}} \\
& \approx\left(\frac{H^{v}\left(P_{0}, t_{k}^{\prime \prime}\right)}{l}\right)^{\frac{\beta_{1}-\beta_{2}}{\beta_{2}}} \\
& =\left(\frac{\operatorname{dist}\left(P_{0}, Q_{0}\right)}{s}\right)^{1-\frac{\beta_{1}}{\beta_{2}}} .
\end{aligned}
$$

where the last equality comes from the definition of $l$.

Hence we obtain the lemma with $\alpha=1-\frac{\beta_{1}}{\beta_{2}}<1$ depending on $\bar{M}$ and $n$.

Let $P_{0} \in \Gamma_{0}$. Since $\Gamma_{t}$ stays Lipschitz in $B_{\bar{r}}\left(P_{0}\right)$, Lemma 2.5 implies that $\Omega_{1}=\Omega_{t\left(P_{0}+s e_{n}\right)}(u) \cap B_{\bar{r}}\left(P_{0}\right)$ satisfies the hypothesis of Lemma 2.6 with $\Omega_{0}=\Omega_{0}(u) \cap B_{\bar{r}}\left(P_{0}\right)$.

Lemma 2.6. Let $\Omega_{i}:=\left\{\left(x^{\prime}, x_{n}\right) \in[-r, r]^{n}: x_{n}<f_{i}\left(x^{\prime}\right)\right\}(i=0,1)$ where $f_{0}$ and $f_{1}$ are Lipschitz functions with a Lipschitz constant less than 1 such that $f_{0}(0)=0,0<f_{1}(0)=s<r / 10$ and for every $x^{\prime} \in\left[-2^{i} s, 2^{i} s\right]^{n-1}$

$$
f_{0}\left(x^{\prime}\right) \leq f_{1}\left(x^{\prime}\right) \leq f_{0}\left(x^{\prime}\right)+a^{i} 2^{i} s
$$

for some $0<a<1$. If $u_{i}(i=0,1)$ is a positive harmonic function in $\Omega_{i}$, which vanishes continuously on $\Gamma_{i}=\left\{\left(x^{\prime}, x_{n}\right): x_{n}=f_{i}\left(x^{\prime}\right)\right\}$, then

$$
u_{1}\left(-s e_{n}\right) \leq C \frac{u_{1}\left((-r / 2) e_{n}\right)}{u_{0}\left((-r / 2) e_{n}\right)} u_{0}\left(-s e_{n}\right)
$$

for $C$ depending only on a and the dimension $n$.

Proof. Denote by $\omega(x, E, \Omega)$ the harmonic measure of a set $E \subset \partial \Omega$ with pole at $x$, that is, the value at $x$ of the harmonic function in $\Omega$ with boundary value 1 on $E$ and 0 on $\partial \Omega \backslash E$.

Without loss of generality, we may assume that

$$
u_{0}\left((-r / 2) e_{n}\right)=u_{1}\left((-r / 2) e_{n}\right)=1 .
$$


Let $v_{0}$ be a harmonic function in $\Omega_{0}$ with boundary data 1 on $\left\{\left(x^{\prime}, x_{n}\right)\right.$ : $\left.x_{n}=-r\right\}$ and 0 elsewhere on its boundary, then by Dahlberg's comparison theorem, $v_{0}\left(-s e_{n}\right) \approx u_{0}\left(-s e_{n}\right)$.

Denote $B_{0}=[-s, s]^{n}$ and $c B_{0}=[-c s, c s]^{n}$ for $c>0$. Let $v_{i}$ be a harmonic function in $\Omega^{i}:=\Omega_{0} \cup\left(\Omega_{1} \cap 2^{i} B_{0}\right)$ such that $v_{i}=1$ on $\left\{\left(x^{\prime}, x_{n}\right)\right.$ : $\left.x_{n}=-r\right\}$ and $v_{i}=0$ elsewhere on its boundary. Then $u_{1}\left(-s e_{n}\right) \approx v_{k}\left(-s e_{n}\right)$ for $k$ such that $[-r, r]^{n} \subset 2^{k} B_{0}$. Hence it suffices to prove the following claim.

Claim. $v_{i}\left(-s e_{n}\right) \leq\left(1+C\left(a^{i \beta_{n} / 2}\right)\right) v_{i-1}\left(-s e_{n}\right)$ where $\beta_{n}>0$ is a dimensional constant.

Proof of Claim. Recall $\Omega^{i}:=\Omega_{0} \cup\left(\Omega_{1} \cap 2^{i} B_{0}\right)$ and observe that $w:=v_{i}-v_{i-1}$ is a positive harmonic function in $D:=2^{i} B_{0} \cap \Omega^{i-1}$ such that $w=0$ on $\partial D \cap 2^{i-1} B_{0}$ and $w=v_{i}-v_{i-1} \neq 0$ on $\partial D-2^{i-1} B_{0}$. Let

$$
E_{1}=\left\{x \in \partial D: \operatorname{dist}\left(x, \partial \Omega_{i}\right) \leq 2^{i} a^{i / 2} s\right\}-2^{i-1} B_{0}
$$

and

$$
E_{2}=\left\{x \in \partial D: \operatorname{dist}\left(x, \partial \Omega_{i}\right)>2^{i} a^{i / 2} s\right\}-2^{i-1} B_{0} .
$$

If $x \in E_{1}$, then

$$
w(x) \leq v_{i}(x) \leq C\left(a^{i / 2}\right)^{\beta_{n}} v_{i}\left(-2^{i} s e_{n}\right) \stackrel{g}{\approx} a^{i \beta_{n} / 2} v_{i-1}\left(-2^{i} s e_{n}\right)
$$

for a dimensional constant $0<\beta_{n}<1$. If $x \in E_{2}$, then

$$
w(x)=v_{i}(x)-v_{i-1}(x) \leq v_{i-1}\left(x-a^{i} 2^{i} s e_{n}\right)-v_{i-1}(x) \leq C a^{i / 2} v_{i-1}(x)
$$

where the first inequality follows from the maximum principle in $D$ and $f_{0} \leq$ $f_{1} \leq f_{0}+a^{i} 2^{i} s$ and the second inequality follows from the following gradient estimate $\left|\nabla v_{i-1}(x)\right| \leq C\left(1 /\left(2^{i} a^{i / 2} s\right)\right) v_{i-1}(x)$ for $x \operatorname{such}$ that $\operatorname{dist}\left(x, \partial \Gamma_{1}\right)>$ $2^{i} a^{i / 2} s$.

From (2.9) and (2.10), we obtain $w\left(-s e_{n}\right) \leq C a^{i \beta_{n} / 2} v_{i-1}\left(-s e_{n}\right)$ since

$$
\omega\left(-s e_{n}, E_{1}, D\right) \approx \omega\left(-s e_{n}, E_{2}, D\right) .
$$

To conclude Case 1, observe that

$$
H\left(P_{0}, T\right) \approx \delta_{0}
$$

since $\Gamma_{t}$ stays Lipschitz in $B_{\bar{r}}\left(P_{0}\right)$. Then by combining Lemmas 2.5 and 2.6, we obtain Theorem 2.1 in Case 1 . 
Case 2 Now it remains to prove the theorem for the general case in which $\Omega$ may not be star-shaped. Let us consider the domain $B_{\bar{r}}\left(P_{0}\right) \times\left[0, t_{0}\right]$ where $t_{0}$ is a positive constant which will be chosen later. In this domain we will trap $u$ between two solutions $\tilde{u}$ and $\tilde{v}$, both of which have starshaped initial positive phase, so that we can apply the result of case 1 .

The main step of the proof is to show that Lemma 2.5 holds for the general case with a constant $C$ in (2.1) depending on $\bar{r}, \bar{R}$ and $n$. Let

$$
t_{0}=\sup \left\{t: \Gamma_{t}(u) \text { is contained in the } \bar{r} / 20 \text {-neighborhood of } \Gamma_{0}(u)\right\} .
$$

Comparing $u$ with radially symmetric supersolution yields that $t_{0}$ is bounded from below by a positive constant depending on $n, \bar{r}$ and $\bar{R}$. Moreover, comparing $u$ with radially symmetric subsolution yields that if we pick a small constant $h$ depending only on $n, \bar{r}$ and $\bar{R}$, then for $P_{0} \in \Gamma_{0}(u)$ and $0<s<\bar{r} h$ we obtain

$$
t_{1}:=t\left(P_{0}+s e_{n}, u\right)<t_{0} .
$$

Note that this lower bound on $t_{0}$ is equivalent to the case $s \approx h \bar{r}$ of Lemma 2.5. It says roughly that when one point of the boundary moves a distance comparable to 1 then so do all the others.

Let $\tilde{\Omega}_{0}$ be starshaped with respect to every $x \in \tilde{K}$ for a sufficiently large ball $\tilde{K}$ in $\tilde{\Omega}_{0}$. Assume that

$$
\begin{gathered}
\Omega_{0}(u) \cap B_{5 \bar{r} / 4}\left(P_{0}\right) \subset \tilde{\Omega}_{0} \cap B_{5 \bar{r} / 4}\left(P_{0}\right) ; \\
\Gamma_{0}(u) \cap B_{\bar{r} / 2}\left(P_{0}\right)=\partial \tilde{\Omega}_{0} \cap B_{\bar{r} / 2}\left(P_{0}\right) ; \\
\Omega_{0}(u) \cap\left(B_{5 \bar{r} / 4}\left(P_{0}\right)-B_{3 \bar{r} / 4}\left(P_{0}\right)\right)+(\bar{r} / 10) e_{n} \subset \tilde{\Omega}_{0} .
\end{gathered}
$$

Let $\tilde{u}$ be a solution to (HS) with a initial positive phase $\Omega_{0}(\tilde{u})=\tilde{\Omega}_{0}-\tilde{K}$ and $\tilde{u}(x, t)=g(t)$ on the fixed boundary $\partial \tilde{K}$. Observe that, by definition of $t_{0}$,

$$
\Omega_{t}(u) \cap\left(B_{5 \bar{r} / 4}\left(P_{0}\right)-B_{3 \bar{r} / 4}\left(P_{0}\right)\right)+(\bar{r} / 40) e_{n} \subset \tilde{\Omega}_{0}, \text { for } 0 \leq t \leq t_{0} .
$$

Therefore if we choose $g(t)$ such that

$$
\tilde{u}\left(P_{0}-(\bar{r} / 4) e_{n}, t\right)=C \text { for } 0 \leq t \leq t_{0},
$$

where $C$ is a dimensional constant, then by Harnack inequality applied to $\tilde{u}(\cdot, t)$ we obtain

$$
u(x, t) \leq 1 \leq \tilde{u}(x, t) \text { for }(x, t) \in\left(\partial B_{\bar{r}}\left(P_{0}\right) \cap \Omega_{t}(u)\right) \times\left[0, t_{0}\right] .
$$


Hence by the comparison principle (Theorem 1.8) applied to $u$ and $\tilde{u}$ in $B_{\bar{r}}\left(P_{0}\right) \times\left[0, t_{0}\right]$, we obtain $u \leq \tilde{u}$ in $B_{\bar{r}}\left(P_{0}\right) \times\left[0, t_{0}\right]$.

Next, we construct a solution $\tilde{v}$ to (HS) with the fixed boundary $\tilde{K}^{\prime} \subset$ $B_{3 \bar{r}}\left(P_{0}\right)$ and the starshaped initial positive phase such that

$$
\begin{aligned}
\Gamma_{0}(\tilde{v}) \cap B_{\bar{r} / 2}\left(P_{0}\right) & =\Gamma_{0}(u) \cap B_{\bar{r} / 2}\left(P_{0}\right) ; \\
\Omega_{0}(\tilde{v}) & \subset \Omega_{0}(u) .
\end{aligned}
$$

By Harnack inequality applied to $u(\cdot, t)$, there is a positive constant $c=c(\bar{r}, \bar{R}, n)<1$ such that

$$
c \leq u(x, t) \text { in } \tilde{K}^{\prime} \times\left[0, t_{0}\right] .
$$

Let us choose the fixed boundary of $\tilde{v}$ by $c$ as given above, so that $\tilde{v} \leq u$ for $x \in \tilde{K}^{\prime}$. Observe that $\tilde{v} \leq 1=u$ for $x \in K$. Therefore by the comparison principle (Theorem 1.8) applied to $u$ and $\tilde{v}$ in $\mathbb{R}^{n}-\left(K \cup \tilde{K}^{\prime}\right) \times\left[0, t_{0}\right]$, we obtain

$$
\tilde{v} \leq u \text { in } B_{\bar{r}}\left(P_{0}\right) \times\left[0, t_{0}\right] .
$$

We remark that, by Harnack inequality applied to $\tilde{v}(\cdot, t)$,

$$
\tilde{v}\left(P_{0}-(\bar{r} / 4) e_{n}, t\right) \stackrel{g}{\approx} 1
$$

It follows from $\tilde{v} \leq u \leq \tilde{u}$ that

$$
H^{\tilde{v}}\left(P_{0}, t_{1}\right) \leq s \leq H^{\tilde{u}}\left(P_{0}, t_{1}\right) .
$$

Also, since $\tilde{v}_{0} \stackrel{g}{\approx} \tilde{u}_{0}$ in $B_{\bar{r} / 4}\left(P_{0}\right)$ and

$$
\tilde{v}\left(P_{0}-(\bar{r} / 4) e_{n}, t\right) \stackrel{g}{\approx} \tilde{u}\left(P_{0}-(\bar{r} / 4) e_{n}, t\right)
$$

for $0 \leq t \leq t_{0}$, it follows that

$$
H^{\tilde{u}}\left(P_{0}, t_{1}\right) \leq C H^{\tilde{v}}\left(P_{0}, t_{1}\right)
$$

for $C$ depending on $\bar{r}, \bar{R}$ and $n$. Hence

$$
s \leq H^{\tilde{u}}\left(P_{0}, t_{1}\right) \leq C s
$$

for $C$ depending on $\bar{r}, \bar{R}$ and $n$.

If $Q_{0} \in \Gamma_{0} \cap B_{\bar{r}}\left(P_{0}\right)$, then by applying Lemma 2.5 for $\tilde{u}$,

$$
H^{u}\left(Q_{0}, t_{1}\right) \leq H^{\tilde{u}}\left(Q_{0}, t_{1}\right) \leq C s\left(\frac{\operatorname{dist}\left(P_{0}, Q_{0}\right)}{s}\right)^{\alpha}
$$


for $C$ depending on $\bar{r}, \bar{R}$ and $n$.

Now we are ready to prove Theorem 2.1. By (2.11), (2.12) and the fact that $\tilde{v} \leq u \leq \tilde{u}$, we obtain

$$
\delta_{0} \leq H^{u}\left(P_{0}, T\right) \leq H^{\tilde{u}}\left(P_{0}, T\right) \leq C H^{\tilde{v}}\left(P_{0}, T\right) \leq C \delta_{0} .
$$

Also by Lemma 2.5, there exists a domain $\Omega^{\prime}$ such that $\Omega_{T}(u) \subset \Omega^{\prime}$ and $\Omega^{\prime} \cap B_{\bar{r}}\left(P_{0}\right)$ is a Lipschitz domain satisfying the hypothesis of Lemma 2.6 with $\Omega_{0}=\Omega_{0}(u) \cap B_{\bar{r}}\left(P_{0}\right)$ and $\Omega_{1}=\Omega^{\prime} \cap B_{\bar{r}}\left(P_{0}\right)$. Since $u(x, T) \leq w(x)$ for the harmonic function $w(x)$ on $\Omega^{\prime}-K$ with data 1 on $\partial K$ and 0 on $\partial \Omega^{\prime}$, we obtain Theorem 2.1 by (2.13) and Lemma 2.6.

Proof of Corollary 2.2 We will use barrier arguments with radially symmetric test functions.

Without the loss of generality, let $P_{2}=P_{0}+s e_{n}$ and $P_{1}=P_{0}-s e_{n}$. Note that the ball $B_{M^{\prime} s}\left(P_{2}\right)$ lies outside of $\Omega_{0}$ for some $M^{\prime}$ depending on $\bar{M}$. We consider $h$ : a radially symmetric solution of (HS) in $B_{2 M^{\prime} s}\left(P_{2}\right)$ with initial domain $B_{2 M^{\prime} s}\left(P_{2}\right)-B_{M^{\prime} s}\left(P_{2}\right)$ and fixed boundary data

$$
h=\sup _{\partial B_{2 M^{\prime} s}\left(P_{2}\right)} u_{0}
$$

Note that by Lemma $1.11 h \leq C s\left|\nabla u_{0}\left(P_{1}\right)\right|$ with $C=C(\bar{M})$, and so $\Gamma_{t}(h)$ moves with speed comparable to $\left|\nabla u_{0}\left(P_{1}\right)\right|$.

By Theorem 2.1, one can choose the constant $C>0$ depending only on $\bar{r}, \bar{R}, \bar{M}$ of the Lipschitz domain $\Omega$ and dimension $n$ such that $u \leq h$ on $\partial B_{2 M^{\prime} \bar{r}}\left(P_{2}\right)$ up to $t=t\left(P_{2}\right)$. Hence it follows that $u \leq h$ up to $t=t\left(P_{2}\right)$. But now it follows that $u\left(P_{2}, \tau\right)=0$ if $h\left(P_{2}, \tau^{\prime}\right)=0$ for $0 \leq \tau^{\prime} \leq \tau$, and $h\left(P_{2}, \tau^{\prime}\right)=0$ if

$$
\tau^{\prime} \leq C s /\left|\nabla u_{0}\left(P_{1}\right)\right| .
$$

Thus it follows that $t\left(P_{2}\right) \geq C s /\left|\nabla u_{0}\left(P_{1}\right)\right|$. To prove the upper bound on $t\left(P_{2}\right)$ one proceed in a parallel way, this time constructing a subsolution of (HS) based on an annulus inside of $\Omega_{0}$.

Proof of Corollary 2.3 Fix $x_{0} \in \Omega_{0}$. Let $r=r_{x_{0}}$ and let $s<r$. Let $B_{i}$ $(i \geq 1)$ be the box having a center at $x_{0}$ and having a side length of $2^{i+1} r$. Denote $B_{0}=\emptyset$ and define

$$
\Omega_{i}=\Omega_{0} \cup\left(\cup_{k=1}^{i}\left(\cup_{p \in\left(B_{k}-B_{k-1}\right) \cap \Gamma_{0}} B\left(p, C\left(\frac{2^{k} r}{s}\right)^{\alpha} s\right)\right)\right)
$$


where $C$ and $0<\alpha<1$ are the constants from Lemma 2.5. Let $v_{i}(x)$ be the harmonic function in $\Omega_{i}$ with boundary values 1 on $\partial K$ and 0 on $\partial \Omega_{i}-\partial K$.

\section{Claim.}

For $i=0,1,2 \ldots$

$$
v_{i+1}\left(x_{0}\right)-v_{i}\left(x_{0}\right) \leq C\left(\frac{s}{r}\right)^{\beta} 2^{-i \beta} u\left(x_{0}, 0\right)
$$

where $v_{0}\left(x_{0}\right)$ denotes $u\left(x_{0}, 0\right), 0<\beta=(1-\alpha) \gamma_{n}<1$ and $0<\gamma_{n}<1$ is a dimensional constant.

We can observe that Corollary 2.3 follows from the claim since Lemma 2.5 implies that for a sufficiently large $i$,

$$
\Omega_{t\left(P_{x_{0}}+s e_{n}\right)} \subset \Omega_{i} \text { and } u\left(x_{0}, t\left(P_{x_{0}}+s e_{n}\right)\right) \leq v_{i}\left(x_{0}\right) .
$$

Proof of Claim.

1. By Lemma 2.6

$$
\omega\left(x_{0}, \partial B_{i}, B_{i} \cap \Omega_{i+1}\right) \leq C \omega\left(x_{0}, \partial B_{i}, B_{i} \cap \Omega_{0}\right) .
$$

2. Let $z$ be a point in the middle of $B_{i+1} \cap \Omega_{0}$, then for $y \in\left(B_{i+1}-\right.$ $\left.B_{i}\right) \cap \partial \Omega_{i}$

$$
\begin{aligned}
v_{i+1}(y) & \leq C \omega\left(y, \partial B_{2^{i} r}(y), B_{2^{i} r}(y) \cap \Omega_{i+1}\right) v_{i+1}(z) \\
& \leq C\left(\frac{\left(\frac{2^{i} r}{s}\right)^{\alpha} s}{2^{i} r}\right)^{\gamma_{n}} v_{i+1}(z) \leq C\left(\frac{s}{2^{i} r}\right)^{(1-\alpha) \gamma_{n}} u(z, 0)
\end{aligned}
$$

where the second inequality follows from $\operatorname{dist}\left(y, \partial \Omega_{i+1}\right) \leq C\left(\frac{2^{i} r}{s}\right)^{\alpha} s$ and the third inequality follows from Lemma 2.6.

3. $v_{i+1}(x)-v_{i}(x)$ is a harmonic function in $\Omega_{i}$ with boundary value $v_{i+1}$ on $\left(B_{i+1}-B_{i}\right) \cap \partial \Omega_{i}$ and 0 elsewhere. Hence

$$
\begin{aligned}
v_{i+1}\left(x_{0}\right) & -v_{i}\left(x_{0}\right) \\
& \leq \omega\left(x_{0}, \partial B_{i} \cap \Omega_{i+1}, B_{i} \cap \Omega_{i+1}\right) \sup _{y \in\left(B_{i+1}-B_{i}\right) \cap \partial \Omega_{i}} v_{i+1}(y) \\
& \leq C \omega\left(x_{0}, \partial B_{i} \cap \Omega_{0}, B_{i} \cap \Omega_{0}\right)\left(\frac{s}{2^{i} r}\right)^{(1-\alpha) \gamma_{n}} u(z, 0) \\
& \leq C\left(\frac{s}{r}\right)^{\beta} 2^{-i \beta} u\left(x_{0}, 0\right)
\end{aligned}
$$

by 1 and 2 . Hence the claim holds with $0<\beta=(1-\alpha) \gamma_{n}<1$. 


\section{The $\epsilon$-monotonicity in space}

We denote by $\alpha(e, f)$ the angle between vectors $e$ and $f$ in $\mathbb{R}^{n}$.

Definition 3.1. $u$ is $\epsilon$-monotone in $D$ for a cone $W(\theta, e)$ if for $\epsilon^{\prime} \geq \epsilon$ and for $x, y-\epsilon^{\prime} e \in D$

$$
u(x) \geq \sup _{y \in B_{\epsilon^{\prime} \sin \theta}(x)} u\left(y-\epsilon^{\prime} e\right) .
$$

Let $u$ be our solution in $\mathbb{R}^{n}, n \geq 3$, given in Theorem 2.1 with a Lipschitz constant less than $a_{n}$ for initial data. Here we will show that the free boundary remains $\epsilon$-monotone in space for small time.

Lemma 3.2. Let $u$ be as given in Theorem 2.1 with Lipschitz constant $\bar{M}<a_{n}$ and $n \geq 3$. Suppose $0 \in \Gamma_{0}$ and let $\theta=\arctan (1 / \bar{M})$ so that

$$
\left(x+W\left(\theta,-e_{n}\right)\right) \cap B_{1}(0) \subset \Omega_{0} ;\left(x+W\left(\theta, e_{n}\right)\right) \cap B_{1}(0) \cap \Omega_{0}=\emptyset
$$

for every $x \in \Gamma_{0} \cap B_{1}(0)$. Then for any $\epsilon>0$ there exists $h>0$ depending on $\epsilon$ and $\bar{M}$ such that $u(\cdot, t)$ is he-monotone for the cone $W\left(\theta-\epsilon,-e_{n}\right)$ in $B_{h}(0)$ for $t \in\left[0, t\left(h e_{n}\right)\right]$.

For the proof Lemma 3.2 we will use Lemma 2.4 and Lemma 3.3 below. Consider a solution $v$ with starshaped initial positive phase $\Omega_{0}(v)$ with respect to all points of $K$. It then follows that $\Gamma_{0}(v)$ is locally Lipschitz with Lipschitz constant $\bar{M}$ depending on the size of $K$, and from Lemma 2.4 that for small time $\Omega_{t}(v)$ is also locally Lipschitz with Lipschitz constant $\bar{M}-\delta$, where $\delta$ depends on the distance between $\Gamma_{t}(v)$ and $\Gamma_{0}(v)$. But now our results in sections 7 to 10 apply and we can conclude that $\Omega(v)$ is indeed smooth in space and time. In particular in the proof of Lemma 3.2 we will be able to compare our original solution $u$ with such functions $v$ as smooth test functions. First we state important properties of $v$ which will be used in the proof of Lemma 3.2.

Lemma 3.3. Let $0 \in \partial \Omega$ and let $\Omega$ be a starshaped region with respect to every $x \in B_{r}\left(-2 e_{n}\right) \subset \Omega$ with $0<r<2$. Fix $0<h<1$ and let $W\left(\theta,-e_{n}\right)$ be the maximal cone such that for any $\zeta \in \Gamma_{0} \cap B_{h}(0), \zeta+W\left(\theta,-e_{n}\right)$ is contained in the smallest cone which has the vertex at $\zeta$ and contains $B_{r}\left(-2 e_{n}\right)$. We also assume that $r$ is chosen large enough such that $\theta>$ $\arctan \left(1 / a_{n}\right)$.

Let $v(x, t)$ be a solution of (HS) with the initial domain $\Omega-B_{r}\left(-2 e_{n}\right)$ and with the fixed boundary data 1 on $\partial B_{r}\left(-2 e_{n}\right)$, then 
(1) there exists $\delta>0$ depending on $h$ such that $v(\cdot, t)$ is monotone in $B_{h}(0)$ for the cone $W\left(\theta-\delta,-e_{n}\right)$ for every $t \leq t\left(h e_{n}\right)$. In particular, $\delta$ tends to 0 as $h$ goes to 0 .

(2) in $B_{h}(0) \times\left[0, t\left(h e_{n}\right)\right]$ the sets

$$
\{v(x, t)=a\},\{v(x, t)=(1+\epsilon) a\},\{v(x,(1+\epsilon) t)=a\}
$$

are contained in the Ch€-neighborhood of each other.

Proof. By Lemma 2.4, $\Omega_{t}(v)$ is starshaped with respect to any point $x_{0} \in$ $B_{r}\left(-2 e_{n}\right)$ and thus (1) is proved. In particular if we choose $h=h(\bar{M})$ small enough then $\Omega$ is Lipschitz in space in $D=B_{h}(0) \times\left[0, t\left(h e_{n}\right)\right]$ with Lipschitz constant $\bar{M}<a_{n}$. We proceed to prove (2) with this choice of $h$.

Due to Lemma 1.11 it follows then that

$$
v\left(x-C h \epsilon e_{n}, t\right) \geq(1+\epsilon) v(x, t) \text { in } D \cap \Omega(u)
$$

for a constant $C>0$ depending only on $\bar{r}, \bar{R}, \bar{M}$ of Lipschitz domain $\Omega$ and dimension $n$. Hence it follows that the sets $\{(x, t): v(x, t)=a\}$ and $\{(x, t): v(x, t)=(1+\epsilon) a\}$ are within distance $C h \epsilon$.

Now it remains to show that the sets $\{(x, t): u(x, t)=a\}$ and $\{(x, t)$ : $v(x,(1+\epsilon) t)=a\}$ are within distance $C h \epsilon$ of each other. For this we need some estimates on $v_{t}$ in $D$. Since $\Omega(u)$ is Lipschitz in space in $D$ with Lipschitz constant $\bar{M}<a_{n}$, our results from section 7 to 10 applies to $u$. In particular due to Corollary 10.9, at each time $t=t(y)$ with $x \in \Gamma_{0}$ and $y=x+\delta e_{n}$,

we have

$$
C_{1}|\nabla v|(y, 0) \leq \frac{v_{t}}{|\nabla v|}(z, t) \leq C_{2}|\nabla v|(y, 0) .
$$

in $z \in B_{\delta}(y) \cap \Omega_{t}(v)$ for constants $C_{1}, C_{2}>0$ depending only on $\bar{r}, \bar{R}, \bar{M}$ of Lipschitz domain $\Omega$ and dimension $n$.

In particular every level set of $v$ moves with normal velocity $V$ less than $C \frac{\delta}{t}$, which is less than $\frac{h}{t}$ if $0<\delta<h$. In particular the level sets $\{v(\cdot, t)=a\}$ and $\{v(\cdot,(1+\epsilon) t=a\}$ are less than $C h \epsilon$-away.

Proof of Lemma 3.2 Let $0 \in \Gamma_{0}$ and let $B_{1}=B_{1}(0)$. We can construct a domain $\Omega^{\prime}$ such that

(i) $\Omega^{\prime} \cap B_{1}=\Omega_{0} \cap B_{1}$; 
(ii) $\Omega^{\prime}$ is star-shaped with respect to every $x \in K^{\prime} \subset \Omega^{\prime}$ for a sufficiently large ball $K^{\prime}$.

Then for the harmonic function $v_{0}$ in $\Omega^{\prime}-K^{\prime}$ with data 1 on $\partial K^{\prime}$ and 0 on $\partial \Omega^{\prime}, v_{0}$ is monotone in $B_{1}$ for the cone $W\left(\theta-\epsilon,-e_{n}\right)$. Also if we let $v(x, t)$ be a solution of (HS) with $v(x, 0)=v_{0}(x)$, then by Lemma 2.4, we may assume that $v(x, t)$ is monotone in $B_{1}$ for the cone $W\left(\theta-2 \epsilon,-e_{n}\right)$ for $t \leq t\left(e_{n}, v\right)$.

To prove the lemma, we will construct a supersolution $w$ and a subsolution $w_{0}$ of (HS) such that in some small ball $B_{h}(0)$,

$$
w_{0} \leq u \leq w
$$

and the free boundaries of $w$ and $w_{0}$ are close to $\Gamma_{(1+C \epsilon) t}(v)$ and $\Gamma_{(1-C \epsilon) t}(v)$ respectively in $B_{h}(0)$. If we show that the level sets of $w$ and $w_{0}$ are also between two Lipschitz graphs within $h \epsilon$-neighborhood of each other, then we can conclude.

Before we define $w$ and $w_{0}$, we construct concentric balls $B_{3} \subset B_{2} \subset B_{1}$ satisfying the properties (3.1), (3.2) and (3.3) below.

Let $B_{2}$ be a concentric ball in $B_{1}$ with the radius of $\epsilon^{k_{0}}$. If $k_{0}$ is sufficiently large, then by Lemma 1.13, a normalization of $v_{0}$ by a suitable constant multiple yields that for any $x \in B_{2} \cap \Omega_{0}(v)=B_{2} \cap \Omega_{0}(u)$

$$
1-\epsilon \leq \frac{u_{0}(x)}{v_{0}(x)} \leq 1+\epsilon
$$

Define

$$
H_{1}:=\Gamma_{0}(v) \cap B_{2}, H_{2}:=\left(\Gamma_{0}(v)-\epsilon^{k_{0}+k_{1}} e_{n}\right) \cap B_{2}
$$

and let $S$ be the region between $H_{1}$ and $H_{2}$.

Let $v_{1}$ be the harmonic function in $B_{2} \cap S$ which has boundary value $v_{0}$ on $H_{2}$ and 0 on elsewhere on its boundary. If $k_{1}$ is sufficiently large, then

$$
1-\epsilon \leq \frac{v_{1}(x)}{v_{0}(x)} \leq 1+\epsilon
$$

for any $x \in\left(\cup_{0 \leq s \leq \epsilon^{k_{0}+k_{1}}}\left(H_{2}+s e_{n}\right)\right) \cap \partial\left(\frac{2}{3} B_{2}\right)$.

Finally, we let $B_{3}$ be a concentric ball in $B_{2}$ with the radius of $\epsilon^{k_{0}+k_{1}+k_{2}}:=$ $h$. Let $t_{0}$ be the time when $\Gamma_{t}(v)$ hits the top of $B_{3}$, i.e. $\Gamma_{t}(v) \cap B_{3}$ moves 
less than $h$ in the $e_{n}$-direction up to the time $t_{0}$. If $k_{2}$ is sufficiently large, then Corollary 2.3 and Lemma 2.6 imply that

$$
\frac{v(x, t)}{v_{0}(x)} \leq 1+\epsilon
$$

for any $x \in H_{2}$ and $t<t_{0}$. Also for large $k_{2} \Gamma_{t_{0}}(v)$ is located between $\Gamma_{0}(v)$ and $\Gamma_{0}(v)+\epsilon^{3 k_{0}} e_{n}$ in $B_{2}$.

Now we construct a supersolution $w$ of (HS) in $\frac{2}{3} B_{2} \times\left[0, t_{0}\right]$. To simplify its construction, translate and rotate to assume that $B_{1}$ has the center at $e_{n} \in \Gamma_{0}(v)$ and $\Gamma_{0}(v)$ is Lipschitz in the direction of $e_{n}$.

Define

$$
\phi(x)=\frac{1}{|x|^{2}} \cdot\left(x_{1}, \ldots, x_{n-1},-x_{n}+2|x|^{2}\right)
$$

where $x=\left(x_{1}, \ldots, x_{n}\right) \in \mathbb{R}^{n}$. Since $\phi$ is the composition of the reflection about $x_{n}=1$ and the conformal mapping sending $x$ to $\frac{x}{|x|^{2}}$, harmonic functions are preserved by $\phi$. The purpose of this conformal mapping is to bend the free boundary up above $\Gamma_{t}(u)$.

Let $y=\phi(x)$ and let $x^{\prime}=\left(x_{1}, \ldots, x_{n-1}\right)$. If $x_{n}=1+m\left|x^{\prime}\right|$, then

$$
\left|y^{\prime}-x^{\prime}\right|=\frac{2 m\left|x^{\prime}\right|^{2}+\left(1+m^{2}\right)\left|x^{\prime}\right|^{3}}{|x|^{2}}
$$

and

$$
y_{n}-x_{n}=\frac{\left(1-m^{2}\right)\left|x^{\prime}\right|^{2}-m\left(1+m^{2}\right)\left|x^{\prime}\right|^{3}}{|x|^{2}} .
$$

Hence for $x=\left(x^{\prime}, x_{n}\right)$ such that $\left|x^{\prime}\right| \leq \epsilon^{k_{0}}$ and $x_{n}=1+m\left|x^{\prime}\right|$ for some $-\bar{M} \leq m \leq \bar{M}<\frac{1}{\sqrt{3}}$,

$$
\left|y^{\prime}-x^{\prime}\right| \leq \frac{1}{\bar{M}}\left(y_{n}-x_{n}\right)
$$

Hence if we further assume $\frac{1}{3} \epsilon^{k_{0}} \leq\left|x^{\prime}\right|$, then

$$
\phi(x) \in W\left(\frac{\pi}{3}, e_{n}\right)+x+\epsilon^{3 k_{0}} e_{n} .
$$

Also we can observe that

$$
\left|y^{\prime}-x^{\prime}\right| \leq 2\left|x-e_{n}\right| \cdot\left|x^{\prime}\right|
$$


and

$$
\left|y_{n}-x_{n}\right| \leq 2\left(\left|x^{\prime}\right|^{2}+\left|x_{n}-1\right|^{2}\right)
$$

This implies

$$
|\phi(x)-x| \leq C\left|x-e_{n}\right|^{2} .
$$

Now for each $t \in\left[0, t_{0}\right]$, we define $w(x, t)$ to be the harmonic function in $B_{2}-\phi\left(\Gamma_{t}(v)\right)-\phi\left(H_{2}\right)$ with boundary data $(1+5 \epsilon) v_{0} \circ \phi^{-1}$ on $\phi\left(H_{2}\right)$ and 0 elsewhere on its boundary.

Since $|\nabla(\phi)-I| \leq C \epsilon^{k_{0}}$ in $B_{2}$ for the identity matrix $I$,

$$
1-C \epsilon^{k_{0}} \leq \frac{\text { normal velocity of } \Gamma_{t}(v) \text { at } x}{\text { normal velocity of } \phi\left(\Gamma_{t}(v)\right) \text { at } \phi(x)} \leq 1+C \epsilon^{k_{0}}
$$

and for $x \in \frac{2}{3} B_{2} \cap\left(\cup_{0 \leq s}\left(H_{2}+s e_{n}\right)\right)$

$$
1-C \epsilon \leq \frac{|D v|(x, t)}{|D w|(\phi(x), t)} \leq 1+C \epsilon
$$

Hence for a sufficiently large $C>0, w(x,(1+C \epsilon) t)$ is a supersolution of (HS) in

$$
D:=\frac{2}{3} B_{2} \cap\left(\cup_{0 \leq s}\left(\phi\left(H_{2}\right)+s e_{n}\right)\right) \times\left[0, t\left(h e_{n}, w\right)\right] .
$$

Next, we show that $u(x, t) \leq w(x,(1+C \epsilon) t)$ on the parabolic boundary of $D$. (3.5) implies that for $x \in H_{2}$

$$
\operatorname{dist}(x, \phi(x)) \leq C \epsilon^{2 k_{0}} .
$$

Hence if we let

$$
\epsilon^{2 k_{0}} \ll \epsilon^{k_{0}+k_{1}} \approx \operatorname{dist}\left(H_{1}, H_{2}\right)
$$

then by (3.1), (3.2) and (3.3)

$$
u(x, t)<(1+5 \epsilon) v_{0}\left(\phi^{-1}(x)\right)
$$

for $x \in \phi\left(H_{2}\right)$ on the fixed boundary of $w$ and for $t<t_{0}$.

Also (3.4) implies that $\Gamma_{t}(w)-\frac{1}{3} B_{2}$ does not intersect $\bar{\Omega}_{t}(u)$ since $B_{3}$ was chosen so that $\Gamma_{t}(v)$ is located between $\Gamma_{0}(v)$ and $\Gamma_{0}(v)+\epsilon^{3 k_{0}} e_{n}$ in $B_{2} \times\left[0, t_{0}\right]$. Hence on $D$

$$
u^{*}(x, t) \leq w(x,(1+C \epsilon) t) .
$$




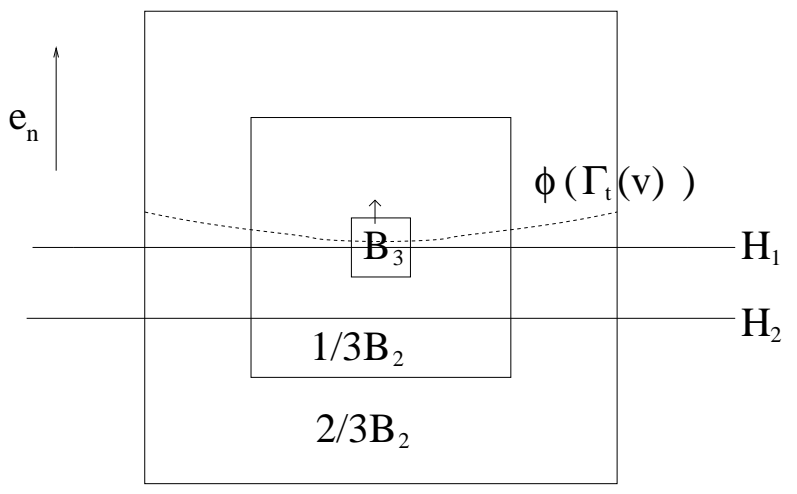

Figure 4.

We construct the subsolution $w_{0}(x,(1-C \epsilon) t)$ in $D$ similarly to $w$. Let

$$
w_{0}=(1-5 \epsilon) v_{0} \circ \bar{\phi}^{-1}
$$

on $\bar{\phi}\left(H_{2}\right)$ where $\bar{\phi}$ is the composition of the reflection about $x_{n}=1$ and the conformal mapping sending $x$ to $\frac{x}{\left|x-2 e_{n}\right|^{2}}$. This conformal mapping bends the free boundary down below $\Gamma_{t}(u)$. Then $w_{0}(x,(1-C \epsilon) t) \leq u(x, t)$.

In $B_{3}, \Gamma_{t}(u)$ is between the free boundaries of $w_{0}(x,(1-C \epsilon) t)$ and $w(x,(1+C \epsilon) t)$, which are contained in the $C h^{2}$ - neighborhoods of $\Gamma_{(1-C \epsilon) t}(v)$ and $\Gamma_{(1+C \epsilon) t}(v)$, respectively.

On the other hand, by (3.5) the level sets

$$
\left\{x \in B_{h}(0): w_{0}(x,(1-C \epsilon) t)=a\right\}
$$

and

$$
\left\{x \in B_{h}(0): w(x,(1+C \epsilon) t)=a\right\}
$$

are between $L_{0}-C h^{2} e_{n}$ and $L_{1}+C h^{2} e_{n}$ where

$$
L_{0}=\left\{x \in B_{h}(0): v(x,(1-C \epsilon) t)=(1+C \epsilon) a\right\}
$$

and

$$
L_{1}=\left\{x \in B_{h}(0): v(x,(1+C \epsilon) t)=(1-C \epsilon) a\right\} .
$$

$L_{0}$ and $L_{1}$ are Lipschitz graphs along the directions in the cone $W(\theta-$ $\left.2 \epsilon,-e_{n}\right)$. Moreover, it follows from the proof of Lemma 2.4 that $L_{0}$ and $L_{1}$ are contained in the $C h \epsilon$-neighborhood of each other. 


\section{Scaled nondegeneracy of $u$ in space variable}

Based on the $\epsilon$-monotonicity of $u$ in space, our next goal is to show that for small time the free boundary stays indeed Lipschitz in space. To show this we will follow the iteration argument used in [C2] improving flatness of the free boundary to Lipschitz, but this procedure requires the nondegeneracy of $u$ on the free boundary, that is, $|\nabla u| \geq c$ on $\Gamma(u)$. We cannot prove this nondegeneracy. Instead we will show that if $u$ is $\epsilon$-monotone in space then $u$ is "nondegenerate at scale $\epsilon$ " (Corollary 4.4).

Proposition 4.1. Let $u$ be a solution of $(H S)$ with $0 \in \Gamma_{0}$. In addition supppose that $u(\cdot, t)$ is $\epsilon$-monotone for $0<\epsilon<\epsilon_{n}$ for the cone $W\left(\theta,-e_{n}\right)$, $\theta>\pi / 4$ in the region $B_{2}\left(e_{n}\right)$ for $t \in\left[0,2 t_{0}\right]$, where $\epsilon_{n}$ is a dimensional constant and $t_{0}=t\left(e_{n}\right)$. Then there exists a dimensional constant $C>0$ such that

$$
B_{1 / 2}\left(e_{n}\right) \cap\left(\Omega_{t}+s e_{n}\right) \subset \Omega_{(1+C \epsilon) t}
$$

for $t_{0} / 2 \leq t \leq t_{0}$ and $0 \leq s \leq \epsilon$.

Proof. For $P_{1} \in B_{1 / 2}\left(e_{n}\right) \cap \Omega_{t}(u)$, denote $t_{1}=t\left(P_{1}\right)$. It suffices to show that $u\left(P_{1}+s e_{n},(1+C \epsilon) t_{1}\right)>0$.

Let $P_{2}=P_{1}+\frac{1}{5} e_{n}, R=B_{1 / 2}\left(P_{2}\right)-B_{1 / 10}\left(P_{2}\right)$ and $\Sigma=R \times\left[0, t_{1}\right]$. Define

$$
w(x, t)=\inf _{B_{\epsilon \varphi(x)}(x)} u\left(y-\epsilon e_{n}, t\right)
$$

where $\varphi$ defined in $R$ satisfies the following properties:

$$
\begin{array}{ll}
\text { (a) } \quad \Delta\left(\varphi^{-Q_{n}}\right)=0 & \text { in } R \\
\text { (b) } \varphi=A_{n} & \text { on } \partial B_{1 / 10}\left(P_{2}\right) \\
\text { (c) } \varphi=1 / \sqrt{2} & \text { in } \partial B_{1 / 2}\left(P_{2}\right) .
\end{array}
$$

Fix $Q_{n}$, a sufficiently large dimensional constant. Then Lemma 9 of [C1] says that $w_{1}$ is superharmonic in $\Omega_{t}(w) \cap R$ for $0 \leq t \leq t_{1}$. Choose $A_{n}$ (depending on $Q_{n}$ ) sufficiently large that $\varphi\left(P_{1}\right)>2$. Note also that $|\nabla \varphi| \leq C$ where $C$ depends on $Q_{n}$ and $A_{n}$.

Now let us compare $w$ and $u$ in $\Sigma$ (see figure 5.) 


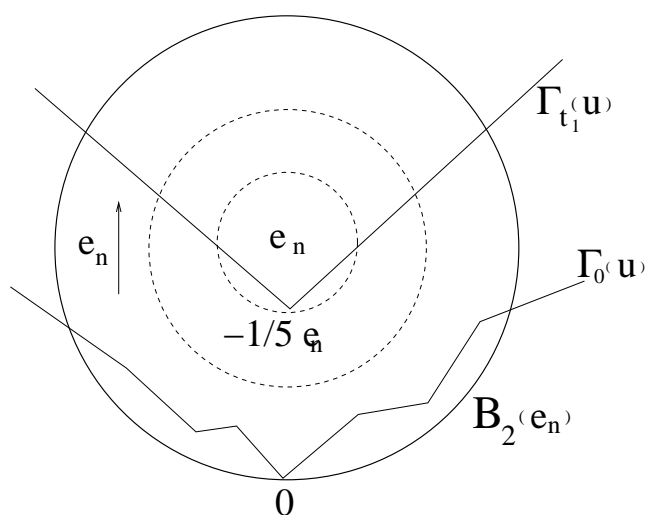

Figure 5.

First observe that due to the $\epsilon$-monotonicity of $u$ (in space), if $\epsilon<1 / 10$, then $w=u=0$ on $\partial B_{1 / 10}\left(P_{2}\right) \times\left[0, t_{1}\right]$ and $\Omega(u)$ lies outside of $B_{1 / 2}\left(P_{2}\right)$ at $t=0$. Moreover, because of the fact $\theta>\pi / 4$ and (c), we have $w \geq u$ on $\partial B_{1 / 2}\left(P_{2}\right) \times\left[0, t_{1}\right]$. Hence $w \geq u$ on the parabolic boundary of $\Sigma$. Thus if we can show that for some constant $C>0$

$$
w_{1}(x, t):=w(x,(1+C \epsilon) t)
$$

is a supersolution of (HS) in $\Sigma$, then by Theorem 1.8 for $u_{\delta}(\cdot, t):=(1-$ $\delta) u(\cdot,(1-\delta) t+\delta), \delta>0$ we have $u_{\delta}^{*} \prec w_{1}$ in $\Sigma \cap\left\{t \geq \delta(1-\delta)^{-1}\right\}$, and in particular $u \leq w_{1}$ in $\Sigma$. On the other hand, since $\varphi\left(P_{1}\right)>2$, $w(x, t) \leq u\left(x+s e_{n}, t\right)$ at $P_{1}$ for $0 \leq s \leq \epsilon$ and thus we can conclude.

To confirm that $w_{1}$ is a supersolution, consider a $C^{2,1}$ function $\psi(x, t)$ such that $w_{1}-\psi$ has a local minimum at $\left(x^{0}, t^{0}\right) \in \Gamma\left(w_{1}\right) \cap \Sigma$ in $\bar{\Omega}\left(w_{1}\right) \cap \Sigma$ with $|\nabla \psi|\left(x^{0}, t^{0}\right) \neq 0$. It would be enough to show that $\psi_{t}-|\nabla \psi|^{2} \geq 0$. For $y^{0}$ such that

$$
u\left(y^{0},(1+C \epsilon) t^{0}\right)=w_{1}\left(x^{0}, t^{0}\right),
$$

the function

$$
u(x+\nu \epsilon \varphi(x),(1+C \epsilon) t)-\psi(x, t), \quad \nu=\frac{y^{0}-x^{0}}{\left|y^{0}-x^{0}\right|}
$$

has a local minimum at $\left(x^{0}, t^{0}\right)$ in $\bar{\Omega}\left(w_{1}\right) \cap \Sigma$. On the other hand

$$
\tilde{u}(x, t):=u(x+\nu \epsilon \varphi(x),(1+C \epsilon) t)
$$

satisfies

$$
\tilde{u}_{t}-|\nabla \tilde{u}|^{2} \geq(1+C \epsilon) u_{t}-|\nabla u|^{2}(1+\epsilon|\nabla \varphi|)^{2} \geq 0
$$


on $\Gamma(\tilde{u})$ (in the viscosity sense) if

$$
C \geq 3 \sup _{B_{\frac{1}{2}}\left(e_{n}\right)-S}|\nabla \varphi| .
$$

For rigorous argument we consider $u(x, t)-\psi_{2}(x, t)$, where $\psi_{2}(x, t)=$ $\psi\left(f^{-1}(x, t)\right)$ and $f(x, t)=(x+\nu \epsilon \varphi(x),(1+C \epsilon) t)$. Then $u-\psi_{2}$ has a local minimum at $f\left(x^{0}, t^{0}\right)$ in $\bar{\Omega}(u) \cap \Sigma$.

Lemma 4.2. (Lemma 2.5, [K2]) Let u be a continuous viscosity solution of $(H S)$ in $S$,

$\left(x_{1}, t_{1}\right) \in \Gamma(u) \cap S$ and let $\phi$ be a $C^{2,1}$-function in a local neighborhood of $\left(x_{1}, t_{1}\right)$ such that $u-\phi$ has a local maximum zero at $\left(x_{1}, t_{1}\right)$ in $\bar{\Omega}(u) \cap\left\{t \leq t_{1}\right\}$ and $|\nabla \phi|\left(x_{1}, t_{1}\right) \neq 0$. Then it follows that

$$
\left(\phi_{t}-|\nabla \phi|^{2}\right)\left(x_{1}, t_{1}\right) \leq 0 \text {. }
$$

Since $|\nabla \psi|\left(x_{0}, t_{0}\right) \neq 0$, the supersolution version of Lemma 4.2 yields that

$$
\left(\left(\psi_{2}\right)_{t}-\left|\nabla \psi_{2}\right|^{2}\right)\left(f^{-1}\left(x^{0}, t^{0}\right)\right) \geq 0 .
$$

After a straightforward computation we obtain

$$
\left(\psi_{t}-|\nabla \psi|^{2}\right)\left(x_{0}, t_{0}\right) \geq 0
$$

and thus $w_{1}$ is a viscosity supersolution of (HS) in $\Sigma$.

Corollary 4.3. If $(x, t) \in \Gamma(u)$, then there exist positive constants $c_{1}$ and $c_{2}$ which depends on $C$ given in (4.1) such that

$$
u\left(\cdot, t /\left(1+c_{1} \epsilon\right)\right)=0 \text { in } B_{c_{2} \epsilon t}(x)
$$

Proof. First suppose $t=t\left(\epsilon_{n} e_{n}\right)$. Then due to Lemma 3.2 and Proposition 4.1 (4.1) will hold for $\tilde{u}$ with $c_{0} / 2 \leq t \leq c_{0}, c_{0}$ depending on $\epsilon_{n}$ and $\bar{M}$. For convenience, we will assume $t=c_{0}$. (The full range $c_{0} / 2 \leq t \leq c_{0}$ follows from the same argument). By rescaling $\tilde{u}(x, t)=a^{-1} u(a x, a t)$, we may assume $c_{0}=1$. If $x \in \Gamma_{1}(u)$, then by Proposition 4.1 one can choose $c_{1}>0$ such that $\left(x-2 \epsilon e_{n}\right) \in \Gamma_{1 /\left(1+c_{1} \epsilon\right)}(u)$. By $\epsilon$-monotonicity of $u$ (Lemma 3.2) one can then choose $c_{2}$ such that

$$
u\left(y, 1 /\left(1+c_{1} \epsilon\right)\right) \leq u\left(x-2 \epsilon e_{n}, 1 /\left(1+c_{1} \epsilon\right)\right)=0
$$


if $y \in B_{c_{2} \epsilon}(x)$.

Corollary 4.4. Let $u, \epsilon, t_{0}$ be as in Lemma 4.1 with $u(0,0)=1$. In addition suppose that $x \in \Gamma_{t}(u) \cap B_{1 / 2}\left(e_{n}\right), \frac{1}{2} t_{0}+\epsilon \leq t \leq t_{0}$ and that there is a spatial ball $B$ of radius $\epsilon$ such that $\bar{B} \cap \bar{\Omega}_{t}=\{x\}$. Then there exists a dimensional constant $C>0$ such that

$$
\sup _{y \in B_{2 \epsilon}(x)} u(y, t) \geq C \epsilon
$$

Proof. Let $c_{1}$ and $c_{2}$ be as given in (3.2). If $u(\cdot, t) \leq C \epsilon$ in $B_{2 \epsilon}(x)$, then $u \leq C \epsilon$ in $B_{2 \epsilon}(x) \times\left(\left(1+c_{1} \epsilon\right)^{-1} t, t\right)$. Then one can compare $u$ with a radially symmetric supersolution of (HS) in $B_{2 \epsilon}(x) \times\left[\left(1+c_{1} \epsilon\right)^{-1} t, t\right]$ to show that for $x$ to be on $\Gamma_{t}(u)$ our constant $C$ cannot be too small.

\section{Lipschitz in the space variable}

In this section we show that the $\epsilon$-monotonicity of $u$ can be improved to the Lipschitz continuity of $u$ via an iteration argument. Here we assume that $a_{n}$ is sufficiently small that (2.0) is satisfied. In the next section we use a special argument for $n=2$ to shos that we can take $a_{2}=1$.

Theorem 5.1. Let $u$ be as given in Theorem 2.1. Moreover assume that $0 \in \Gamma_{0}(u), u\left(-e_{n}, 0\right)=1$ and $u(\cdot, t)$ is $\epsilon$-monotone for a cone $W\left(\theta,-e_{n}\right)$ in $B_{2}\left(e_{n}\right)$ for $0 \leq t \leq t\left(e_{n}\right)$. If $\pi / 2-\theta<\tan ^{-1}\left(a_{n}\right)$ and $0<\epsilon<1 / 100$ then there is a constant $0<\lambda<1$ depending only on $\bar{r}, \bar{R}, \bar{M}$ of Lipschitz domain $\Omega$ and dimension $n$ such that $u(\cdot, t)$ is $\lambda \epsilon$-monotone for $W\left(\theta^{\prime},-e_{n}\right)$ in $B_{2-\epsilon^{1 / 4}}\left(e_{n}\right)$ for $\epsilon^{1 / 6} \leq t \leq t\left(e_{n}\right)$ with $\theta^{\prime}=\theta-\epsilon^{1 / 14}$.

Corollary 5.2. Let $u$ be given as in Theorem 5.1. Then there is a constant $\epsilon_{1}>0$ depending only on $\bar{r}, \bar{R}, \bar{M}$ of Lipschitz domain $\Omega$ and dimension $n$ such that if $0<\epsilon<\epsilon_{1}$, then $u(\cdot, t)$ is fully monotone in every direction of the cone $W\left(\theta^{\prime},-e_{n}\right)$ in $B_{1 / 2}\left(e_{n}\right)$ for $t \in\left[\frac{1}{2} t\left(e_{n}\right), t\left(e_{n}\right)\right]$ where $\theta^{\prime}=\theta-O\left(\epsilon^{1 / 14}\right)$.

Proof. By iterating Theorem 5.1, we obtain that $u(\cdot, t)$ is monotone in the cone $W\left(\theta^{\prime},-e_{n}\right)$ in $B_{a}\left(e_{n}\right)$ for $\left[b, t\left(e_{n}\right)\right]$, where

$$
a=1-\epsilon^{1 / 4} \Sigma_{k=0}^{\infty} \lambda^{k / 4} \quad ; \quad b=\epsilon^{1 / 6} \Sigma_{k=0}^{\infty} \lambda^{k / 6} .
$$

and

$$
\theta^{\prime}=\theta-\epsilon^{1 / 14} \Sigma_{k=0}^{\infty} \lambda^{k / 14}
$$


Now we turn to the proof of Theorem 5.1. We first prove the result for $t_{0} / 2<t<t_{0}$ and then use a scaling argument to prove the lemma up to $t=\epsilon^{1 / 6}$. The nondegeneracy of $u$ obtained in Corollary 4.4 allows us to adapt the method developed by Caffarelli [C1] and [C2]. The following lemma is due to Caffarelli.

Lemma 5.3. ([C2]) Let $u(x)$ be $\epsilon$-monotone for $W(\theta, e)$ and let

$$
v(x)=\sup _{B_{\zeta(x)}(x)} u(y) .
$$

Assume that $|\nabla \zeta|<1$ and

$$
\sin \bar{\theta} \leq \frac{1}{1+|\nabla \zeta|}\left(\sin \theta-\frac{\epsilon}{2 \zeta} \cos ^{2} \theta-|\nabla \zeta|\right) .
$$

Then $v$ is fully monotone for $W(\bar{\theta}, e)$.

Next we introduce a family of radius functions which is a slight modification of those constructed in Lemma 3.2 of [K2].

Lemma 5.4. For a given constant $C_{0}>0$ there exist constants $k, C^{\prime}>0$ such that for sufficiently small $r, h>0$ and for $0<\eta<1$ there exists a $C^{2}$ function $\varphi(x, t)$ defined in

$$
D:=\left[B_{1}\left(e_{n}\right)-B_{1 / 8}\left(\frac{1}{4} e_{n}\right)\right] \times(r / 2, r)
$$

such that

(a) $1 \leq \varphi \leq 1+\eta h$ in $D$,

(b) $\varphi \Delta \varphi \geq C_{0}|\nabla \varphi|^{2}$ holds in $D$,

(c) $\varphi \equiv 1$ outside $B_{4 / 9}\left(e_{n}\right) \times(3 r / 5, r)$,

(d) $\varphi \geq 1+k \eta h$ in $B_{1 / 3}\left(e_{n}\right) \times(3 r / 4, r)$,

(e) $|\nabla \varphi| \leq C^{\prime} \eta h$ and $0 \leq(\varphi)_{t} \leq C^{\prime} \eta h / r$ in $D$.

Proposition 5.5. Let $u(\cdot, t)$ be a solution of $(H S)$ in $B_{1}(0) \times\left[t\left(e_{n}, u\right),-t\left(e_{n}, u\right)\right]$ with

$$
C \epsilon^{1 / 8} \leq t\left(e_{n}, u\right) \leq 1
$$


where $C>0$ is a constant depending only on $\bar{r}, \bar{R}, \bar{M}$ of Lipschitz domain $\Omega$ and dimension $n$. Moreover suppose that $u(\cdot, t)$ is $\epsilon$-monotone for the cone $W\left(\theta,-e_{n}\right)$ with $\pi / 2-\theta<\tan ^{-1}\left(a_{n}\right)$ for $|t| \leq t\left(e_{n}, u\right)$.

Then there exists a constant $\epsilon_{0}>0$ and $0<\lambda<1$ depending only on $\bar{r}, \bar{R}, \bar{M}$ of Lipschitz domain $\Omega$ and dimension $n$ such that if $\epsilon^{\prime}<\epsilon_{0}$ then $u(\cdot, t)$ is $\lambda \epsilon^{\prime}-$ monotone in

$$
W\left(\theta^{\prime}, e_{n}\right), \theta^{\prime}=\theta-O\left(\epsilon^{1 / 7}\right)
$$

in $B_{\frac{1}{3}}\left(e_{n}\right)$ for $t \in\left[\frac{3}{4} t\left(e_{n}, u\right), t\left(e_{n}, u\right)\right]$.

Proof of Proposition 5.5: By definition of $a_{n}$, if $\epsilon<d<<1$ then

$$
C_{1} d^{7 / 6} \leq u\left(x-d e_{n}, t\right) \leq C_{2} d^{5 / 6}
$$

for $(x, t) \in \Gamma(u) \cap B_{1}(0) \times\left[0, t\left(e_{n}\right)\right]$. Let $t_{0}:=t\left(e_{n}, u\right)$ and consider

$$
v(x, t):=\sup _{y \in B_{\sigma \epsilon \varphi(x, t)}} u(y, t) \quad \text { in } B_{1 / 2}\left(e_{n}\right) \times\left[t_{0} / 2, t_{0}\right]
$$

where $\sigma=[\sin \theta-(1-\lambda)]$ for $1-\sin \pi / 4<\lambda<1$. Here $\varphi$ is the test function constructed in Lemma 5.4 with $r=t_{0} / 4$. As in Lemma 9 of [C1], the dimensional constant $C_{0}$ is chosen sufficiently large so that $v$ is subharmonic.

Due to (5.1) and Lemma 5.4(e) $\left|\varphi_{t}\right| \leq C \epsilon^{-1 / 8}$.

Lemma 5.6. (see Definition 1.5)

$$
\Gamma_{t}\left(v^{*}\right)=\Gamma_{t}(v) .
$$

Proof. The reason why we can show $\Gamma_{t}\left(v^{*}\right)=\Gamma_{t}(v)$ when the corresponding statement for $u$ cannot yet be proved is that (by Lemma 5.3) $v(\cdot, t)$ is fully monotone in a cone of directions.

It follows from a simple barrier argument that $\Gamma_{t}\left(u^{*}\right)$ satisfies the following no-jump condition, namely, for any $x \in \Gamma_{t}\left(u^{*}\right)$ there is $t_{n}<t$ and $x_{n} \in \Gamma_{t_{n}}\left(u^{*}\right)$ such that $x_{n} \rightarrow x$ and $t_{n} \rightarrow t$. Hence the same holds for

$$
v^{*}(x, t):=\sup _{y \in B_{\sigma \epsilon \varphi(x, t)}} u^{*}(y, t) .
$$

Note that by Theorem 1.8 we have 


$$
u(x, t) \leq u^{*}(x, t) \leq u(x, t+\epsilon) \text { for any } \epsilon>0
$$

Since $\varphi$ increases in time it follows that

$$
v(x, t) \leq v^{*}(x, t) \leq v(x, t+\epsilon) \text { for any } \epsilon>0 .
$$

Suppose that $x_{0} \in \Gamma_{t_{0}}(v)$ and $x_{0} \notin \Gamma_{t_{0}}\left(v^{*}\right)$. Then $B_{r}\left(x_{0}\right) \subset \Omega_{t_{0}}\left(v^{*}\right)$ for some $r>0$. Hence $x_{1} \in \Gamma_{t_{0}}\left(v^{*}\right)$ with $x_{1}=x_{0}+h e_{n}, h \geq r$. On the other hand, by (5.3) and the full monotonicity of $v(\cdot, t), v^{*}(x, t)=0$ in $B_{h / 10}\left(x_{1}\right)$ for $t<t_{0}$. This violates the property of $\Gamma_{t}\left(v^{*}\right)$. Next suppose that $x_{0} \in \Gamma_{t_{0}}\left(v^{*}\right)$ and $x_{0} \notin \Gamma_{t_{0}}(v)$. Then $v\left(x, t_{0}\right)=0$ for $\left|x-x_{0}\right|<r$ for some $r>0$. Hence by (5.4), $v^{*}(x, t)=0$ for $\left|x-x_{0}\right|<r, t<t_{0}$. This again violates the no-jump condition of $\Gamma_{t}\left(v^{*}\right)$.

For any $\delta>0$ and for any set $S \in \mathbb{R}^{n}$, let

$$
N_{t}(s)=\left\{y \in \mathbb{R}^{n}: d\left(y, \Gamma_{t}(v)\right)<s\right\}
$$

and

$$
N(s)=\left\{(x, t): x \in N_{t}(s)\right\} .
$$

For $t_{0} / 2 \leq t \leq t_{0}$, let $w(\cdot, t)$ be the harmonic function defined in the domain

$$
N_{t}\left(M \epsilon^{5 / 7}\right) \cap \Omega_{t}(v) \cap B_{1}(0),
$$

with boundary data $v$ on $\partial N_{t}\left(M \epsilon^{5 / 7}\right) \cap \Omega_{t}(v)$ and zero elsewhere on the boundary. Note that $\Gamma_{t}(v)$ is Lipschitz graph and moves continuously in time by Lemma 5.6. Lemma 5.6 implies $w^{*}(\cdot, t)=0$ on $\Gamma_{t}\left(w^{*}\right)=\Gamma_{t}(w)=\Gamma_{t}(v)$ for each $t>0$.

From now on we write $C$ as a positive constant depending only on $\bar{r}, \bar{R}, \bar{M}$ and $n$. Our plan is to compare $\bar{v}_{\delta}(x, t)=\bar{v}(x, t-\delta)$, where $\bar{v}=v^{*}+C \epsilon^{3 / 7} w^{*}$ with $u_{1}(x, t):=u\left(x-\lambda \epsilon e_{n}, t\right)$ in the domain

$$
\Sigma=N\left(M \epsilon^{5 / 7}\right) \cap\left(B_{1 / 2}\left(e_{n}\right) \times\left[t_{0} / 2, t_{0}\right]\right) .
$$

for arbitrarily small $\delta>0$. We will first show that $\bar{v}_{\delta} \leq u_{1}$ on the parabolic boundary of $\Sigma$. Then we prove that $\bar{v}_{\delta}$ is a viscosity subsolution of (HS) in $\Sigma$. Theorem 1.8 then yields that $\bar{v}_{\delta} \leq u_{1}$ in $\Sigma$. Proposition 5.5 will then follow by evaluating $\varphi$ in the region $B_{1 / 3}\left(e_{n}\right) \times\left[\frac{3}{4} t\left(e_{n}\right), t\left(e_{n}\right)\right]$ using Lemma 
5.4 (d) and by sending $\delta \rightarrow 0$ and using the lower semicontinuity of $u$ to obtain

$$
\bar{v} \leq u \text { in } \Sigma \text {. }
$$

Observe that along

$$
L_{1}:=\partial N\left(M \epsilon^{4 / 7}\right) \cap \Omega(u) \cap\left(B_{3 / 4}\left(e_{n}\right) \times\left(t_{0} / 2, t_{0}\right]\right)
$$

we have, for $l_{1}<l_{2}<\lambda \sin \theta$,

$$
\sup _{B_{l_{1}}(x)} u(y, t) \leq \sup _{B_{l_{2}}(x)} u(y, t)-C\left(l_{2}-l_{1}\right)\left|\nabla u_{1}\right|(x, t) .
$$

Observe that due to (5.1) we can choose constants $C_{1}>C_{2}>0$ depending only on $\bar{r}, \bar{R}, \bar{M}$ of Lipschitz domain $\Omega$ and dimension $n$ such that both level sets $\left\{u=C_{i} M \epsilon^{5 / 6}\right\}, i=1,2$ lies between $N_{t}(M \epsilon)$ and $N_{t}\left(M \epsilon^{5 / 7}\right)$. Since $u(\cdot, t)$ is fully monotone in $W\left(\theta,-e_{n}\right) M \epsilon$-away from $\Gamma_{t}(u)$, we can apply Lemma 1.11 to $u-C M \epsilon^{5 / 6}$ and obtain

$$
\left|\nabla u_{1}\right| \geq \frac{u_{1}}{C \epsilon^{5 / 7}} \quad \text { on } L_{1} .
$$

Thus we obtain from (5.6)

$$
\sup _{B_{l_{1}}(x)} u(y, t) \leq\left[1-\frac{l_{2}-l_{1}}{C \epsilon^{5 / 7}}\right] u_{1}(x, t) .
$$

Hence if we choose $\eta$ in the definition of $\varphi$ such that

$$
[\sin \theta-(1-\lambda)](1+\eta h) \leq \lambda \sin \theta-C \epsilon^{1 / 7},
$$

we obtain $v \leq\left(1-C \epsilon^{3 / 7}\right) u_{1}$ on $L_{1}$.

Note that by definition of $\varphi$ and by the $\epsilon$-monotonicity of $u$,

$$
v \leq u_{1} \text { outside } B_{4 / 9}\left(e_{n}\right) \times\left(3 t_{0} / 4, t_{0}\right) .
$$

Thus by applying Dahlberg's Lemma to $\epsilon^{3 / 7} w$ and $u_{1}-v$ on the region $L_{2}:=N\left(M \epsilon^{4 / 7}\right) \cap \Omega(v) \cap\left(B_{3 / 4}\left(e_{n}\right) \times\left(t_{0} / 2, t_{0}\right)-B_{4 / 9}\left(e_{n}\right) \times\left(\frac{3}{4} t_{0}, t_{0}\right)\right)$, 
we obtain $C \epsilon^{3 / 7} w \leq u_{1}-v$ on $L_{2}$. Therefore $\bar{v}_{\delta} \leq u_{1}$ on the parabolic boundary of $\Sigma$, which is contained in the union of $L_{1}$ and $L_{2}$.

Next we prove that $\bar{v}$, and therefore $\bar{v}_{\delta}$ is a subsolution of (HS) in $\Sigma$. Suppose that $\bar{v}-\psi$ has a local maximum at $\left(x_{1}, t_{1}\right) \in \Gamma(\bar{v})$ in $\bar{\Omega}(\bar{v}) \cap B_{1 / 2}\left(e_{n}\right) \times\left[t_{0} / 2, t_{0}\right]$. Observe that due to Lemma $1.10 w \leq C v$. Therefore $v^{*}-\left(1-C \epsilon^{3 / 7}\right) \psi$ has a local maximum at $\left(x_{1}, t_{1}\right) \in \Gamma(v)$ in $\bar{\Omega}(v) \cap\left[t_{0} / 2, t_{0}\right]$.

By definition of $v^{*}$, there is a point $y_{1} \in \Gamma_{t_{1}}\left(u_{1}\right)$ such that

$$
y_{1}=\bar{\Omega}_{t_{1}}\left(u_{1}\right) \cap \bar{B}_{\epsilon \sigma \varphi\left(x_{1}, t_{1}\right)}\left(x_{1}\right)
$$

and

$$
v(x, t) \geq u_{1}(f(x, t))
$$

where $f$ is defined as

$$
f(x, t):=(x+\nu \epsilon \sigma \varphi(x, t), t) ; \quad \nu=y_{1}-x_{1} /\left|y_{1}-x_{1}\right| .
$$
where

Hence $u_{1}^{*}-\tilde{\psi}$ has a local maximum zero at $\left(y_{1}, t_{1}\right)$ in $\bar{\Omega}\left(u_{1}\right) \cap\left\{s \leq t_{1}\right\}$

$$
\tilde{\psi}(y, s):=\left(1-C \epsilon^{3 / 7}\right) \psi\left(f^{-1}(y, s)\right) .
$$

Due to the properties of $\varphi$ one can easily verify that $f$ is invertible and each component of $f^{-1}$ is $C^{2,1}$ in a neighborhood of $\left(y_{1}, t_{1}\right)$. Therefore $\tilde{\psi}$ is $C^{2,1}$ in space-time in a neighborhood of $\left(y_{1}, t_{1}\right)$.

Note that by definition of $\bar{v}, \epsilon^{3 / 7} w^{*}-\psi$ also has a local maximum at $\left(x_{1}, t_{1}\right)$. Due to Corollary 4.4,

$$
|\nabla \psi|\left(y_{1}, t_{1}\right) \geq \epsilon^{3 / 7}|\nabla w| \geq C \epsilon^{3 / 7} .
$$

Hence $|\nabla \tilde{\psi}| \neq 0$ and we can apply Lemma 4.2 to yield

$$
\tilde{\psi}_{t}-|\nabla \tilde{\psi}|^{2} \leq 0
$$

A straightforward computation using (5.9), the definition of $\tilde{\psi}$ and the upper bounds of $|\nabla \varphi|,\left|\varphi_{t}\right|$ with $r=t_{0} \geq \epsilon^{1 / 8}$ leads to

$$
\psi_{t}-C \epsilon^{7 / 6}|\nabla \psi|^{2} \leq\left(1+C \epsilon^{3 / 7}\right)^{2}|\nabla \psi|^{2} \text { at }\left(x_{1}, t_{1}\right) .
$$

Rearranging terms, we obtain 


$$
\psi_{t}-|\nabla \psi|^{2} \leq C \epsilon^{3 / 7}|\nabla \phi|\left(C \epsilon^{7 / 8-3 / 7}-|\nabla \psi|\right) \leq 0 \text { at }\left(x_{1}, t_{1}\right)
$$

if $\epsilon$ is sufficiently small, where the last inequality is due to (5.8)

Now that we have shown that for all $\delta>0, \bar{v}_{\delta} \leq u_{1}$ in all of $\Sigma$, Proposition 5.5 follows from (5.5).

Proof of Theorem 5.1: Consider a point $\left(x_{0}, t_{0}\right)$ in

$$
\Gamma(u) \cap\left\{B_{2-\epsilon^{1 / 4}}\left(e_{n}\right) \times\left(\epsilon^{1 / 6}, t\left(e_{n}, u\right)\right\}\right.
$$

and let

$$
u_{h}(x, t):=h u\left(\epsilon^{1 / 4} x+x_{0}, \frac{\epsilon^{1 / 2}}{h} t+t_{0}\right) .
$$

where $h=\left[u\left(x_{0}-\epsilon^{1 / 4} e_{n}, t_{0}\right)\right]^{-1}$. By definition of $a_{n}$, if $\epsilon<d<<1$ then

$$
C_{1} d^{7 / 6} \leq u\left(x-d e_{n}, t\right) \leq C_{2} d^{5 / 6}
$$

for $(x, t) \in \Gamma(u) \cap B_{1}(0) \times\left[0, t\left(e_{n}, u\right)\right]$. By construction of $u_{h}$ we have $u_{h}\left(-e_{n}, 0\right)=1$ and $u_{h}$ is $\epsilon^{\prime}=\epsilon^{3 / 4}$ - monotone in the cone $W\left(\theta, e_{n}\right)$ in $B_{1}(0) \times\left[-t\left(e_{n}, u_{h}\right), t\left(e_{n}, u_{h}\right)\right]$.

Note that $u\left(-e_{n}, t\right)=C u\left(-e_{n}, 0\right)=C$ for $-t\left(e_{n}, u\right) \leq t \leq t\left(e_{n}, u\right)$ by Theorem 2.1. Hence it follows from (5.10) and the $\epsilon^{\prime}$-monotonicity of $u_{h}$ it follows that

$$
1 \leq u_{h}\left(-e_{n}, t\right) \leq C \epsilon^{5 / 24-7 / 24}=C \epsilon^{-1 / 12}=C \epsilon^{\prime-1 / 8}
$$

for $-t\left(e_{n}, u_{h}\right) \leq t \leq t\left(e_{n}, u_{h}\right)$. (The first inequality holds because $u_{h}$ increases in time.)

Due to (5.11) and the $\epsilon^{\prime}$-monotonicity of $u_{h}$ one can check from a barrier argument with a radially symmetric barrier that (5.1) holds for $u_{h}$, i.e.,

$$
C \epsilon^{\prime 1 / 8} \leq t\left(e_{n}, u_{h}\right) \leq 1
$$

Hence Proposition 5.5 applies to $u_{h}(x, t)$. In terms of $u$, the proposition states that $u(x, t)$ is $\lambda \epsilon$-monotone for the cone $W\left(\theta^{\prime},-e_{n}\right)$ in

$$
B_{\epsilon^{1 / 2} / 2}\left(x_{0}\right) \times\left[t_{0}, t_{0}+\epsilon^{19 / 32} t\left(e_{n}, u\right)\right] .
$$


with $\theta^{\prime}=\theta-O\left(\epsilon^{3 / 28}\right)$. Therefore $u$ is $\lambda \epsilon$-monotone for the cone $W\left(\theta^{\prime}, e_{n}\right)$ in the region

$$
B_{1-\epsilon^{1 / 4}}(0) \times\left[\epsilon^{1 / 6}, t\left(e_{n}, u\right)\right] .
$$

Theorem 5.7. Let $u$ be as given in Theorem 2.1. In addition suppose that there is $m>0$ such that at each $x \in \Gamma_{0} u(\cdot, 0)$ is monotone for the cone $W(\theta, \nu), \nu=\nu(x)$ in $B_{m}(x)$. If $\pi / 2-\theta<a_{n}$, then there is $t_{0}=$ $t_{0}(\bar{m}, \bar{M}, n)>0,0<h<1$ and $\theta^{\prime}>\pi / 2-\tan ^{-1}\left(2 a_{n}\right)$ such that for any $x \in \Gamma_{0}, \Gamma_{t}(u)$ is monotone for the cone $W\left(\theta^{\prime}, \nu\right)$ in $B_{m / 2}(x)$ for $t \in\left[0, t_{0}\right]$.

Proof. Fix $x_{0} \in \Gamma_{0}$ and let $\nu=-e_{n}$ without loss of generality. Due to Lemma 3.2 , if $\bar{M}$ is sufficiently small then there are positive constants $0<$ $h<1$ and $\epsilon<\epsilon_{1}$ such that for $m^{\prime}=h m, u(\cdot, t)$ is $m^{\prime} \epsilon$-monotone in the cone $W\left(\theta,-e_{n}\right)$ in $B_{m^{\prime}}\left(x_{0}\right)$ for $t \in\left[0, t\left(x_{0}+m^{\prime} e_{n}\right)\right]$, where $\pi / 2-\theta$ is sufficiently small and $\epsilon_{n}$ is a unit vector depending on $x_{0}$.

Define

$$
\tilde{u}(x, t)=h u\left(m^{\prime} x+x_{0}, h\left(m^{\prime}\right)^{2} t\right)
$$

where $h=\left[u\left(x_{0}-m^{\prime} e_{n}, 0\right)\right]^{-1}$. Then it follows from Corollary 5.2 that $\tilde{u}(\cdot, t)$ is Lipschitz for the cone $W\left(\theta^{\prime},-e_{n}\right), \theta^{\prime}=\theta-O\left(\epsilon^{1 / 14}\right)$ in $B_{1 / 2}\left(e_{n}\right)$ with $\frac{1}{2} t\left(e_{n}, \tilde{u}\right) \leq t \leq t\left(e_{n}, \tilde{u}\right)$. In terms of $u$ this implies that $u(\cdot, t)$ is Lipschitz for the cone $W\left(\theta^{\prime},-e_{n}\right)$ in $B_{1 / 2 m^{\prime}}\left(x_{0}\right)$ with $t\left(x_{0}+m^{\prime} e_{n}\right) / 2 \leq t \leq t\left(x_{0}+m^{\prime} e_{n}\right)$.

Now if we repeat step 1 with replacing $x_{0}$ with any $y_{0} \in B_{m / 2}\left(x_{0}\right)$ and $u$ with the scaling

$$
\tilde{u}=h^{-1} u\left(a x+y_{0}, h a^{2} t\right)
$$

where $h=\left[u\left(a x+y_{0}, 0\right)\right]^{-1}$ and $a$ is any positive constant between 0 and $m^{\prime}$, it follows that $u(\cdot, t)$ is Lipschitz for the cone $W\left(\theta^{\prime},-e_{n}\right)$ in $B_{m / 2}\left(x_{0}\right)$ with $0 \leq t \leq t\left(x_{0}+m^{\prime} e_{n}\right)$.

Finally observe that, by Corollary 2.2,

$$
t\left(x_{0}+m^{\prime} e_{n}, u\right) \geq t_{0}(\bar{m}, L, n)>0 .
$$

Remark 1. Our hypothesis in Theorem 5.7 holds if, for example, $\Gamma_{0}$ is $C^{1}$.

2. If $\Gamma(u)$ is Lipschitz in space at each time, it is not hard to check that $\Gamma_{t}(u)$ changes continuously in time in terms of the Hausdorff distance, and hence $u$ is continuous due to Theorem 1.9 (b). In particular $u=u^{*}$. 


\section{Lipschitz in space, $n=2$}

For $n=2$ a much simpler argument using reflection yields the conclusion of Theorem 5.7 under the weaker hypothesis that the Lipschitz constant $\bar{M}<a_{2}=1$.

Theorem 6.1. For $P_{0} \in \Gamma_{0}$, suppose that $u_{0}$ is monotone for the cone $W\left(\theta,-e_{2}\right), \pi / 4<\theta \leq \pi / 2$ in $\Omega_{0} \cap B_{h}\left(P_{0}\right)$ for small $h=C(\bar{M}) \bar{r}$. Then for $0 \leq \tau \leq a=\frac{1}{4}(\theta-\pi / 4), u(\cdot, t)$ is monotone for the cone $W\left(\theta-\tau,-e_{2}\right)$ in $\Omega_{t} \cap B_{a h / 10}(0)$ for $0 \leq t \leq t_{0}$, where

$$
t_{0}=t_{0}(\tau):=\inf _{x \in B_{h}\left(P_{0}\right) \cap \Gamma_{0}} t\left(x+\tau^{k} h e_{2}\right)
$$

with $k(\bar{r}, \bar{R}, \bar{M})>1$.

Proof. The hypothesis on $u_{0}$ in the theorem follows from Lemma 1.11. After rescaling by $u_{h}(x, t):=u\left(h x, h^{2} t\right)$, we may assume $P_{0}=0$ and $h=1$ and $r>2$. Consider a unit vector $p \in \mathbb{R}^{2}$ which has angle $\theta-\tau$ with $-e_{2}$. To prove the theorem we must show that $u$ is monotone increasing in the direction of such vector $p$ in $B_{a / 10}(0) \times\left[0, t_{0}\right]$.

For a unit vector $p$ in $\mathbb{R}^{2}$, define

$$
l_{p}=\left\{v \in \mathbb{R}^{2}: v \cdot p=0\right\} .
$$

Divide $B_{1}(0)$ into two parts $B^{+} \cup B^{-}$, where

$$
B_{1}^{+}=B_{1}(0) \cap\left\{v \in \mathbb{R}^{2}: v \cdot p \geq 0\right\} ; \quad B^{-}=B_{1}(0)-B^{+} .
$$

Also let $l_{p}=l_{p}^{+} \cup l_{p}^{-}$where

$$
l_{p}^{+}:=\left\{v \in l_{p}: v \cdot e_{2} \geq 0\right\}, \quad l_{p}^{-}:=l_{p}-l_{p}^{+} .
$$

(see Figure 5.) Note that $l_{p} \cap \partial B_{1}(0)$ is more than $a$-away from $\Gamma_{0}, l_{p}^{+} \cap$ $B_{1}(0) \subset \Omega_{0}$ and $l_{p}^{-} \cap B_{1}(0) \subset \mathbb{R}^{2}-\Omega_{0}$.

Define $w(\cdot, t)$ as the reflection of $u(\cdot, t)$ with respect to $l_{p}$. We will show that $u \leq w$ in $\Sigma:=B^{-} \times\left[0, t_{0}\right]$ by comparing these functions on the parabolic boundary of $\Sigma$.

Observe that in $B^{-} \times\left[0, t_{0}\right], w$ is a viscosity solution of (HS) with $w \geq u$ at $t=0$ and $w=u$ on $l_{p}$. Moreover by definition of $t_{0}$, for $0 \leq t \leq t_{0}$ $u(\cdot, t)=0$ on

$$
S_{1}:=B^{-} \cap \partial B_{1}(0) \cap\left\{x \in \mathbb{R}^{2}: d\left(x, \Omega_{0}\right)>\tau\right\} .
$$




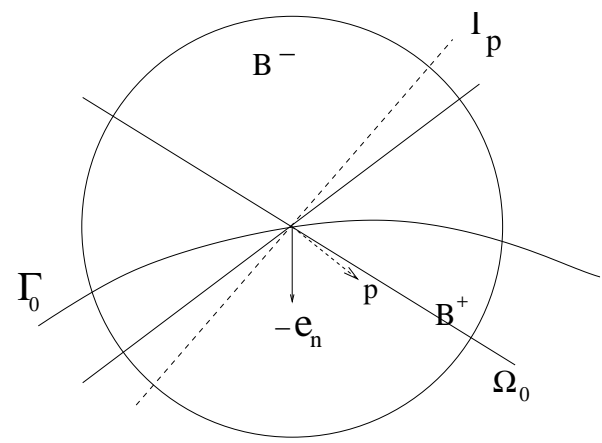

Figure 6.

Hence $u=0 \leq \omega$ on $S_{1} \times\left[0, t_{0}\right]$.

Therefore to apply Theorem 1.8, it remains to prove that $u(\cdot, t) \leq w(\cdot, t)$ on $S_{2}:=\partial B^{-}-S_{1}$ for $0 \leq t \leq t_{0}$. Divide $S_{2}$ into two parts:

$$
S_{2,1}=S_{2} \cap\left\{x: d\left(x, \Gamma_{0}\right) \leq c_{0} a\right\} \text { and } S_{2,2}=S_{2}-S_{2,1}
$$

where $c_{0}$ is a small constant depending only on $\bar{r}, \bar{R}, \bar{M}$ which will be chosen later. Then Theorem 2.1 combined with Lemma 1.10 and from a barrier argument with a radially symmetric solution, it follows that for sufficiently small $c_{0}$ and for $k>1$ in the definition of $t_{0}$ we have

$$
u(x, t) \leq u(x+2 a p, 0) \text { if }(x, t) \in S_{2,1} \times\left[0, t_{0}\right] .
$$

Also note that

$$
w(x, t)=u(x-2(p \cdot x) p, t) \geq u(x+4 a p, 0) \text { in } S_{2,1} \times\left[0, t_{0}\right]
$$

since $-p \cdot x \geq 2 a$ on $S_{2,1}$. Hence for $0 \leq t \leq t_{0}$ and for $x \in S_{2,1}$

$w(x, t)-u(x, t) \geq u(x+4 a p, 0)-u(x, t) \geq u(x+2 a p, t)-u(x+2 a p, 0) \geq 0$.

3. Finally to show that $w \leq u$ in

$$
S_{2,2}=B^{-} \cap \partial B_{1}(0) \cap\left\{x \in \Omega_{t}: d\left(x, \Gamma_{0}\right)>c_{0} a\right\}
$$

we will apply Lemma 1.12. We only need to show that $u$ is $a \epsilon_{0^{-}}$monotone increasing in the direction of $p$ for $\epsilon_{0}=c_{0} \epsilon_{1}$ with a uniform constant $\epsilon$ in the domain $D \times\left(0, t_{0}\right)$, where $D=B_{2}(0) \cap\left\{x \in \Omega_{t}: d\left(x, \Gamma_{0}\right)>c_{0} a\right\}$. To 
check this, note that if $k$ is sufficiently big in the definition of $t_{0}$, then for $t \leq t_{0}$

$$
\begin{aligned}
u\left(x+a \epsilon_{0} p, t\right)-u(x, t) & \geq u\left(x+a \epsilon_{0} p, 0\right)-u(x, t) \\
& \geq C a \epsilon_{1} \tau|\nabla u(x, 0)|-u(x, t)+u(x, 0) \geq 0
\end{aligned}
$$

(third inequality holds if $k$ is big enough to apply Corollary 2.3).

Above argument implies that $\omega \geq u$ in $B^{-} \times\left[0, t_{0}\right]$ and thus $u$ is monotone increasing along $p$ on $l_{p} \cap B_{1}(0)$. The same argument follows for shifts of $l_{p}$ by distance $a / 10$ in the direction $e_{2}$ and $-e_{2}$. Hence $u$ is monotone increasing along $p$ in the domain

$$
\Sigma=\cup\left\{l_{p}+\epsilon e_{2}:|\epsilon| \leq a / 10\right\} \times\left[0, t_{0}\right],
$$

which contains $B_{a / 10}(0) \times\left[0, t_{0}\right]$.

\section{Lower bound on the speed of free boundary}

From this section we only consider $t \in\left[0, t_{0}\right]$, where $\left[0, t_{0}\right]$ is the time interval where $\Gamma_{t}$ stays Lipschitz in space.

Theorem 7.1. Let $P_{0} \in \Gamma_{0}, \bar{M}=\operatorname{Lip}\left(\Gamma_{0} \cap B_{8 l}\left(P_{0}\right)\right)<a_{n}$. Then there exists a constant $C>0$ depending only on $\bar{r}, \bar{R}, \bar{M}$ of Lipschitz domain $\Omega$ and dimension $n$ such that for any $0<\epsilon<1$

$$
u\left(x+l \epsilon e_{n},(1+C \epsilon) t\right)>0
$$

if $x \in B_{2 l}\left(P_{0}\right) \cap \Omega_{t}$ and $t\left(P_{0}+l e_{n}\right) \leq t \leq t\left(P_{0}+2 l e_{n}\right)$.

Proof. By Theorem 5.7 and Theorem 6.1, $\Gamma(u)$ is Lipschitz in space. Hence Proposition 4.1 applies with arbitrarily small $\epsilon>0$ and (7.1) follows.

In particular, Theorem 7.1 implies that the normal speed of the free boundary at $P_{0}+l e_{n}$ multiplied by $C$ is bounded below by $\frac{l}{t\left(P_{0}+l e_{n}\right)}$, the average normal speed of travel from $P_{0}$ to $P_{0}+l e_{n}$, which, in turn, is comparable to $\left|\nabla u_{0}\right|\left(P_{0}-l e_{n}\right)$ by Corollary 2.2. This can be also reformulated as follows. 
Corollary 7.2. For $P_{0} \in \Gamma_{0}$ and $t\left(P_{0}+l e_{n}\right) \leq t \leq t\left(P_{0}+2 l e_{n}\right)$, we have

$$
|\nabla u(x, t)| \geq C\left|\nabla u_{0}\left(P_{0}-l e_{n}\right)\right| \text { for almost every } x \in \Gamma_{t} \cap B_{3 l}\left(P_{0}\right)
$$

where $\nabla u$ is taken as the nontangential limit from $\Omega_{t}(u)$ and $C$ is a constant depending only on $\bar{r}, \bar{R}, \bar{M}$ and $n$.

Proof. The nontangential limit of $\nabla u(\cdot, t)$ from the positive set $\Omega_{t}(u)$ exists almost everywhere since $\Omega_{t}(u)$ is a Lipschitz domain. Fix $t\left(P_{0}+l e_{n}\right) \leq t \leq$ $t\left(P_{0}+2 l e_{n}\right)$ and $x \in \Gamma_{t}(u)$. For any small $\delta>0$ consider $y=x+\delta e_{n}$. Then Theorem 7.1 applies with $\epsilon=\delta / l$ and we obtain

$$
t(y)-t(x) \leq C \frac{\delta}{l} t\left(P_{0}+l e_{n}\right)
$$

By Corollary 2.2 we have

$$
\left|\nabla u\left(x-\delta e_{n}, t\right)\right| \stackrel{g}{\approx} \frac{\delta}{t(y)-t(x)}
$$

and Corollary 7.2 follows since

$$
\frac{l}{t\left(P_{0}+l e_{n}\right)} \stackrel{g}{\approx}\left|\nabla u\left(P_{0}-l e_{n}\right)\right| .
$$

\section{Interior upper bound on speed}

Lemma 8.1. Let $\Phi$ be a conformal mapping from $\mathbb{R}_{+}^{2}:=\{(x, y): y>0\}$ to $D:=\{(x, y): y>f(x)\}$ with $\Phi(\infty)=\infty$, where $f$ is a Lipschitz function on $\mathbb{R}$ with Lipschitz constant less than 1 . Let $d s=\left|\Phi^{\prime}(x, 0)\right| d x=(1 / h) d x$, then

$$
\frac{1}{\int_{J} d s} \int_{J} h^{3} d s \leq C\left(\frac{1}{\int_{J} d s} \int_{J} h d s\right)^{3}
$$

for any interval $J \subset \partial D$.

Proof. Since $\left|\arg \left(\Phi^{\prime}\right)^{2}\right| \leq c_{0}<\pi / 2$, the theorems of Helson and Szegö [HS] and Hunt, Muckenhoupt and Wheeden [HMW] imply

$$
\left(\frac{\int_{I}\left|\Phi^{\prime}\right|^{2} d x}{\int_{I} d x}\right)\left(\frac{\int_{I}\left|\Phi^{\prime}\right|^{-2} d x}{\int_{I} d x}\right) \leq C .
$$


Since $d x=h d s,(8.1)$ can be stated as

$$
\int_{J} h^{-1} d s \int_{J} h^{3} d s \leq C\left(\int_{J} h d s\right)^{2}
$$

where $J=\Phi(I)$.

By the Cauchy Schwarz inequlaity,

$$
\left(\int_{J} d s\right)^{2} \leq\left(\int_{J} h d s\right)\left(\int_{J} h^{-1} d s\right)
$$

Now combine (8.2) and (8.3) to obtain

$$
\left(\int_{J} h^{3} d s\right)\left(\int_{J} d s\right)^{2} \leq C\left(\int_{J} h d s\right)^{3} .
$$

Lemma 8.2. Let $\Omega_{0}$ be a Lipschitz domain with Lipschitz constant $\bar{M}<a_{n}$ and containing a compact set $K$ with a smooth boundary. Let $\Gamma=\partial \Omega_{0}$, $\Omega=\Omega_{0}-K(\partial \Omega=\Gamma \cup \partial K)$ and let $u$ be harmonic in $\Omega$ with boundary values 1 on $\partial K$ and 0 on $\Gamma$. Then there are global constants $0<c_{1}, C_{1}<\infty$ such that the following holds.

(a) The nontangential limit

$$
h(\zeta)=\lim _{z \rightarrow \zeta}|\nabla u(z)|, z \in T_{\zeta}:=\{z \in \Omega: \operatorname{dist}(z, \zeta)<2 \operatorname{dist}(z, \Gamma)\}
$$

exists for almost every $\zeta \in \Gamma$ with respect to surface measure $d \sigma$.

(b) Fix $z_{0} \in \Omega$ at a fixed distance from $\partial K$ and $\Gamma$, then the harmonic measure $d \omega^{z_{0}}$ for $\Omega$ satisfies

$$
C_{1}^{-1} h d \sigma \leq d \omega^{z_{0}} \leq C_{1} h d \sigma .
$$

(c) The nontangential maximal function

$$
h^{\star}(\zeta):=\sup _{z \in T_{\zeta}}|\nabla u(z)|
$$


belongs to $L^{3}(\Gamma, d \sigma)$ and $L^{2}\left(\Gamma, d \omega^{z_{0}}\right)$. In particular,

$$
v(z):=\int_{\Gamma} h(\zeta)^{2} d \omega^{z}(\zeta)
$$

is well-defined and is the harmonic function with nontangential boundary values $h^{2}$ on $\Gamma$ and 0 on $\partial K$.

(d) $|\nabla u(z)|^{2} \leq C_{1}(v(z)+u(z))$.

(e) $v(z) \leq C_{1}|\nabla u(z)|^{2}$ for every $z \in \Omega$ such that $\operatorname{dist}(z, \Gamma) \leq c_{1}$.

Proof. Parts (a) and (b) follow from Dahlberg's theorem [D] and regularity of the Dirichlet problem [Theorem 4.13, JK3]. Part (c) follows for sufficiently small Lipschitz constant from Dahlberg [D2]. In the case $n=2$, we showed in Lemma 8.1 that for Lipschitz constant less than $1, d \omega$ is in the weight class $B_{3}(d \sigma)$. The property that $h \in L^{2}\left(d \omega^{z}\right)$ is equivalent to $h \in L^{3}(d \sigma)$ since $d \omega \approx h d \sigma$. By Dahlberg's theorem, $h^{\star} \in L^{3}(d \sigma)$ [Corollary 3.2, JK3] and hence $h \in L^{2}(d \omega)$. The existence of the nontangential limit of $v$ is a Fatou-type theorem of Hunt and Wheeden [Theorem 2.3", JK3].

To prove part $(\mathrm{d})$, let $\delta=\operatorname{dist}(z, \Gamma)$ and let

$$
H_{1}=\int_{B_{2 \delta}(z)} h d \sigma \approx \frac{u(z)}{\delta} \sigma\left(B_{2 \delta}(z)\right) .
$$

Furthermore, $d \omega^{z} \approx \frac{1}{H_{1}} h d \sigma$ on $B_{2 \delta}(z)$ [JK3, Lemma 2.7]. Therefore

$$
v(z)=\int_{\Gamma} h^{2} d \omega^{z} \gtrsim \frac{1}{H_{1}} \int_{B_{2 \delta}(z)} h^{3} d \sigma \geq \frac{H_{1}^{2}}{\sigma\left(B_{2 \delta}(z)\right)^{2}} \approx\left(\frac{u(z)}{\delta}\right)^{2} \approx|\nabla u(z)|^{2}
$$

where the second inequality follows from Hölder's inequality.

To prove part (e) requires the localized estimate

$$
\frac{1}{\sigma\left(B_{r}\right)} \int_{B_{r}} h^{3} d \sigma \leq\left(\frac{C}{\sigma\left(B_{r}\right)} \int_{B_{r}} h d \sigma\right)^{3}
$$

proved in Lemma 8.1 for $n=2$ and Lipschitz constant $\bar{M}<1$, and proved in [HMW] for $n \geq 3$ and sufficiently small Lipschitz constant (see also Theorem $8.2(\mathrm{~b}))$. 
Let

$$
\delta=\operatorname{dist}(z, \Gamma)=|z-\zeta| \text { for some } \zeta \in \Gamma .
$$

Let $B_{k}=B_{2^{k} \delta}(\zeta)$ and choose $z_{k} \in B_{k}$ such that $\operatorname{dist}\left(z_{k}, \Gamma\right) \approx \delta 2^{k}$. Denote $H_{k}=\int_{B_{k} \cap \Gamma} h d \sigma$, then

$$
\begin{gathered}
\frac{d \omega^{z}}{d \omega^{z_{k}}}(\zeta) \approx \frac{u(z)}{u\left(z_{k}\right)} \text { for } \zeta \in \Gamma-B_{k} \text { and } z \in B_{k}, \\
d \omega^{z_{k}}(\zeta) \approx \frac{1}{H_{k}} h d \sigma(\zeta) \text { for } \zeta \in B_{k+1} \cap \Gamma
\end{gathered}
$$

and

$$
\frac{H_{k}}{\sigma\left(B_{k}\right)} \approx \frac{u\left(z_{k}\right)}{2^{k} \delta}
$$

Hence

$$
\begin{aligned}
v(z)=\int_{\Gamma}|\nabla u|^{2} d \omega^{z} & \approx \sum_{k} \int_{B_{k+1}-B_{k}} h^{2}(\zeta) \frac{u(z)}{u\left(z_{k}\right)} \frac{h(\zeta)}{H_{k}} d \sigma(\zeta) \\
& \leq \sum_{k} \int_{B_{k+1}} h^{3} d \sigma \frac{u(z)}{u\left(z_{k}\right) H_{k}} \\
& \leq \sum_{k} \frac{u(z)}{u\left(z_{k}\right)} \frac{H_{k}^{2}}{\sigma\left(B_{k}\right)^{2}} \\
& \leq u(z) \sum_{k} \frac{u\left(z_{k}\right)}{\left(2^{k} \delta\right)^{2}}
\end{aligned}
$$

where the second inequality follows from (8.4) and the third inequality follows from (8.5).

For sufficiently small Lipschitz constant $(\bar{M}<1$ for $n=2)$, $u$ vanishes more slowly than quadratically. In other words, for some $a>0$,

$$
C u(z) \geq\left(\frac{\operatorname{dist}(z, \Gamma)}{\operatorname{dist}\left(z_{k}, \Gamma\right)}\right)^{2-a} u\left(z_{k}\right)=2^{(2-a) k} u\left(z_{k}\right) .
$$

Thus

$$
u(z) \sum_{k} \frac{u\left(z_{k}\right)}{\left(2^{k} \delta\right)^{2}} \leq C \frac{u(z)^{2}}{\delta^{2}} \approx|\nabla u(z)|^{2} .
$$


Lemma 8.3. Let $\bar{M}<a_{n}$. Then there exists a constant $C>0$ depending only on $\bar{r}, \bar{R}, \bar{M}$ and dimension $n$ such that

$$
u_{t+}(z, t):=\limsup _{\epsilon \rightarrow 0^{+}} \frac{u(z, t+\epsilon)-u(z, t)}{\epsilon} \leq C|\nabla u(z, t)|^{2}
$$

for $z \in \Omega_{t}$ such that $d\left(z, \Gamma_{t}\right) \leq 1 / C$.

Proof. Throughout the proof we denote $C$ as various positive constants depending only on $\bar{r}, \bar{R}, \bar{M}$ and $n$. Let $z \in \Omega_{t}$. By definition, there is a sequence $\epsilon_{m}>0, m=1,2, .$. such that

$$
u_{t+}(z, t)=\lim _{m \rightarrow \infty} \frac{u\left(z, t+\epsilon_{m}\right)-u(z, t)}{\epsilon_{m}} .
$$

By Corollary 2.2, we can choose $\delta_{m}(y)$ depending on $\epsilon_{m}$ and $y$ such that

$$
\max _{B_{2 \delta_{m}(y)}(y)} u(\cdot, t) \stackrel{g}{\approx} \frac{\delta_{m}(y)^{2}}{\epsilon_{m}} .
$$

Define

$$
w_{m}(z, t)=\frac{u\left(z, t+\epsilon_{m}\right)-u(z, t)}{\epsilon_{m}} .
$$

Since Theorem 2.1 implies

$$
\max _{B_{2 \delta_{m}(y)}(y)} u\left(\cdot, t+\epsilon_{m}\right) \stackrel{g}{\approx} \frac{\delta_{m}(y)^{2}}{\epsilon_{m}}
$$

we obtain

$$
\max _{B_{2 \delta_{m}(y)}(y)} w_{m}(\cdot, t) \leq C \frac{\delta_{m}(y)^{2}}{\epsilon_{m}^{2}} \stackrel{g}{\approx}|\nabla u(\tilde{z}, t)|^{2}
$$

where $\tilde{z} \in \Omega_{t} \cap B_{2 \delta_{m}(y)}(y)$ is at least $\delta_{m}(y)$-away from $\Gamma_{t}$ and the approximation follows from the remark below Corollary 2.2. Let

$$
G_{m}(y)=\left\{z \in B_{2 \delta_{m}(y)}(y) \cap \Omega_{t}: \operatorname{dist}\left(z, \Gamma_{t}\right)>\delta_{m}(y)\right\} .
$$

Then for every $z \in \cup_{y \in \Gamma_{t}} G_{m}(y)$,

$$
w_{m}(z, t) \leq C|\nabla u(z, t)|^{2} \leq C(v(z, t)+u(z, t)) \leq C\left[v(z, t)+\max _{y}\left(\delta_{m}(y)\right)^{1 / 2}\right]
$$

where the second inequality follows from Lemma 8.2 (d). 
On $\partial K, w_{m}(z, t)=0$. Hence by maximum principle for harmonic functions,

$$
w_{m}(\cdot, t) \leq C\left[v(\cdot, t)+\max _{y}\left(\delta_{m}(y)\right)^{1 / 2}\right]
$$

in $\Omega_{t}-\cup_{y \in \Gamma_{t}} G_{m}(y)$. Let $\epsilon_{m} \rightarrow 0$, then $\max _{y} \delta_{m}(y) \rightarrow 0$ and for all $z \in \Omega_{t}$,

$$
u_{t+}(z, t) \leq C v(z, t) .
$$

Finally, Lemma 8.2 (e) gives

$$
u_{t+}(z, t) \leq C|\nabla u(z, t)|^{2} .
$$

for $z$ close to $\Gamma_{t}$.

\section{Propagation of the gain to the free boundary}

By Lemma 8.3 and Theorem 2.1, $u$ is Lipschitz continuous, in space and time respectively, away from the free boundary. Over the next two sections we will show that this interior estimates propagate to the free boundary at later times. Ultimately the free boundary will be regular enough that $\nabla u$ exists up to the free boundary and the free boundary equation $V=|\nabla u|$ holds in the classical sense for $t>0$. We use the iteration process introduced by Caffarelli ([ACS], [C1]) with modifications necessitated by the lack of a uniform upper bound on $|\nabla u|$ up to the free boudnary.

The proof of the following lemma is the same as that of Lemma 2.6 in [K2].

Lemma 9.1. Let $u$ solve $(H S)$ in $B_{1}(0) \times[-r, r]$. Assume that $(0,0) \in \Gamma(u)$, $|\nabla u|\left(-\frac{3}{4} e_{n}, 0\right)=1$ and $\Gamma(u)$ is Lipschitz in space with Lipschitz constant $M<a_{n}$. In addition suppose that there exist constants $\delta>0$ and $0 \leq A \leq$ $B, \mu:=B-A$ such that

$$
\alpha\left(\nabla u,-e_{n}\right) \leq \delta \text { and } A \leq \frac{u_{t}}{-e_{n} \cdot \nabla u} \leq B
$$

in $B_{1 / 6}\left(-\frac{3}{4} e_{n}\right) \times(-\delta / \mu, \delta / \mu)$ with $\frac{\delta}{\mu}<r$. Then there exist a unit vector $\nu \in \mathbb{R}^{n}$ and positive constants $r_{0}, b_{0}<1$ depending only on $M, B$ and $n$ such that

$$
\alpha(\nabla u(x, t), \nu) \leq b_{0} \delta \text { in } B_{1 / 8}\left(-\frac{3}{4} e_{n}\right) \times\left(-r_{0} \delta / \mu, r_{0} \delta / \mu\right)
$$


Next we introduce a family of test functions.

Lemma 9.2. (Lemma 3.2, [K2]) For a given constant $C_{0}>0$ there exist constants $k, C^{\prime}>0$ such that for sufficiently small $r, h>0$ and for $0<\eta<1$ there exists a $C^{2}$ function $\varphi_{\eta}(x, t)$ defined in

$$
D:=\left[B_{1}(0)-B_{1 / 8}\left(-\frac{3}{4} e_{n}\right)\right] \times(-r, r)
$$

such that

(a) $1 \leq \varphi_{\eta} \leq 1+\operatorname{r\eta h}$ in $D$,

(b) $\varphi_{\eta} \Delta \varphi_{\eta} \geq C_{0}\left|\nabla \varphi_{\eta}\right|^{2}$ holds in $D$,

(c) $\varphi_{\eta} \equiv 1$ outside $B_{8 / 9}(0) \times\left(-\frac{7}{8} r, r\right)$,

(d) $\varphi_{\eta} \geq 1+r k \eta h$ in $B_{1 / 2}(0) \times\left(-\frac{1}{2} r, r\right)$,

(e) $\left|\nabla \varphi_{\eta}\right| \leq C^{\prime} \eta h$ and $0 \leq\left(\varphi_{\eta}\right)_{t} \leq C^{\prime} \eta h$ in $D$.

Lemma 9.3. Let u satisfy the hypotheses of Lemma 9.1. In addition suppose that

$$
|\nabla u| \geq m_{0}>0 \text { in } \Omega(u) \cap\left(B_{1}(0) \times(-r, r)\right) .
$$

Then there exist a unit vector $\nu_{1} \in \mathbb{R}^{n}$ and a constant $0<\bar{c}<1$ depending on $M, m_{0}$ and $B$ such that

$$
\alpha\left(\nabla u(x, t), \nu_{1}\right) \leq \delta_{1} \text { in } \Omega(u) \cap\left(B_{1 / 2}(0) \times(-\delta / B, \delta / B)\right)
$$

where $\delta_{1} \leq \delta-\bar{c} \frac{\delta^{2}}{\mu}$.

Before proving Lemma 9.3, we first show the following lemma.

Lemma 9.4. Let $u_{1}, u_{2}$ be viscosity solutions of (HS) with $\left|\nabla u_{1}\right| \geq m_{0}$ in $B_{1}(0) \times(-r, r)$. Suppose $\Gamma\left(u_{2}\right)$ is Lipschitz in space in $B_{1}(0) \times(-r, r)$ with Lipschitz constant $M<a_{n}$ and $(0,0) \in \Gamma\left(u_{2}\right)$. In addition suppose

$$
v_{\epsilon}(x, t):=\sup _{y \in B_{\epsilon}(x)} u_{1}(y, t) \leq u_{2}(x, t) \text { in } B_{1}(0) \times(-r, r) .
$$


Then for given $\kappa, \sigma>0$ there exists $0<\epsilon_{0}, h_{0}, c_{0}<1$ depending only on $m_{0}, M$ and $\kappa$ such that if

$$
\begin{aligned}
& u_{2}(x, t)-v_{(1+r \sigma h) \epsilon}(x, t) \geq \kappa \sigma \epsilon u_{2}\left(-\frac{3}{4} e_{n}, t\right) \text { in } B_{1 / 8}\left(-\frac{3}{4} e_{n}\right) \times(-r, r) \\
& \text { for } 0<\epsilon<\epsilon_{0} \text { and } 0<h<h_{0} \text { then }
\end{aligned}
$$

$$
v_{\left(1+c_{0} r \sigma h\right) \epsilon}(x, t) \leq u_{2}(x, t) \text { in } B_{1 / 2}(0) \times(-r / 2, r) .
$$

Proof. As before $C$ represents positive constants depending only on $M$ and $m_{0}$. Define

$$
v(x, t)=v_{\epsilon \varphi_{\sigma}(x, t)}(x, t),
$$

where $\varphi_{\sigma}$ is as defined in Lemma 9.2. Let $w(x, t):=w^{t}(x)$ to satisfy

$$
\begin{cases}-\Delta w^{t}(x)=0 & \text { for }(x, t) \in D \cap \Omega\left(u_{2}\right) ; \\ w=0 & \text { in }\left\{u_{2}=0\right\} \cup\left(\partial B_{1}(0) \times(-r, r)\right) ; \\ w=u_{2}\left(-\frac{3}{4} e_{n}, t\right) & \text { on } \partial B_{1 / 8}\left(\frac{3}{4} e_{n}\right) \times(-r, r) .\end{cases}
$$

Note that by $(9.2),(9.3)$ and the maximum principle of harmonic functions

$$
v+\kappa \sigma \epsilon w \leq u_{2} \text { in } D-D^{\prime}, D^{\prime}=\left[B_{8 / 9}(0) \times\left(-\frac{7}{8} r, r\right)\right] \cap D .
$$

On the other hand, by Lemma $1.10, w \geq C u_{2}$ in $D^{\prime}$. Thus it follows that $\tilde{v}:=(1+C \kappa \sigma \epsilon) v \leq u_{2}$ on the parabolic boundary of $D^{\prime}$.

Next we prove that, for small $\epsilon$ and $h, \tilde{v}$ is a viscosity subsolution of (HS) in $D^{\prime}$. Suppose that there is a $C^{2,1}$ function $\psi(x, t)$ such that $\tilde{v}-\psi$ has a local maximum zero at $\left(x_{1}, t_{1}\right)$ in $\bar{\Omega}(v) \cap\left\{t \leq t_{1}\right\} \cap D^{\prime}$. Since $v$ is subharmonic, we only have to consider the case $\left(x_{1}, t_{1}\right) \in \Gamma(v)$. By definition of $v$, there is a point $y_{1} \in \Gamma_{t_{1}}\left(u_{1}\right)$ such that

$$
y_{1}=\bar{\Omega}_{t_{1}}\left(u_{1}\right) \cap \bar{B}_{\epsilon \varphi_{\sigma}\left(x_{1}, t_{1}\right)}\left(x_{1}\right)
$$

and

$$
v(x, t) \geq u_{1}(f(x, t))
$$

where $f$ is defined as 


$$
f(x, t)=\left(x+\nu \epsilon \varphi_{\sigma}(x, t), t\right) ; \quad \nu=\frac{y_{1}-x_{1}}{\left|y_{1}-x_{1}\right|} .
$$

Hence $u_{1}-\tilde{\psi}$ has a local maximum zero at $\left(y_{1}, t_{1}\right)$ in $\bar{\Omega}\left(u_{1}\right) \cap\left\{s \leq t_{1}\right\}$ where $\tilde{\psi}(y, s):=(1-C \kappa \sigma \epsilon) \phi\left(f^{-1}(y, s)\right)$. By the properties of $\varphi_{\sigma}$ one can easily verify that $f$ is invertible and each component of $f^{-1}$ is $C^{2,1}$ in a neighborhood of $\left(y_{1}, t_{1}\right)$. Therefore $\tilde{\psi}$ is $C^{2,1}$ in space-time in a neighborhood of $\left(y_{1}, t_{1}\right)$.

Moreover since $u_{1}\left(\cdot, t_{1}\right) \leq \tilde{\psi}\left(\cdot, t_{1}\right)$ in $\bar{\Omega}_{t_{1}}\left(u_{1}\right)$ in a neighborhood of $y_{1}$ and with $u_{1}\left(y_{1}, t_{1}\right)=\tilde{\psi}\left(y_{1}, t_{1}\right)$,

$$
|\nabla \tilde{\psi}|\left(y_{1}, t_{1}\right) \geq\left|\nabla u_{1}\right|\left(y_{1}, t_{1}\right) \geq m_{0}>0 .
$$

Hence Lemma 4.2 applies and we obtain

$$
\tilde{\psi}_{t} \leq|\nabla \tilde{\psi}|^{2} \text { at }\left(y_{1}, s_{1}\right) \text {. }
$$

Next a straightforward computation leads to

$$
\psi_{t}-C h \sigma \epsilon|\nabla \psi| \leq(1-C \kappa \sigma \epsilon)(1+C h \sigma \epsilon)^{2}|\nabla \psi|^{2} \text { at }\left(x_{1}, t_{1}\right) .
$$

Rearranging terms, we obtain

$$
\psi_{t}-|\nabla \psi|^{2} \leq \sigma \epsilon|\nabla \psi|[(C h-C \kappa)|\nabla \psi|+C h+O(\epsilon)] \leq 0 \text { at }\left(x_{1}, t_{1}\right)
$$

if $\epsilon<<h$ and $h \leq C \kappa m_{0}$.

Thus $\tilde{v}$ is a viscosity subsolution of (HS) and we can apply Theorem 1.8 to $\tilde{v}(x, t)$ and $u_{2}\left(x+\epsilon e_{n}, t\right)$ in $D^{\prime}$ for every $\epsilon>0$ to yield that

$$
\tilde{v} \leq u_{2} \text { in } D^{\prime},
$$

which yields our assertion by evaluating $\varphi_{\sigma}$ in $D^{\prime}$.

\section{Proof of Lemma 9.3:}

We use the notation of Lemma 9.4. First observe that Lemma 9.1 yields constants $0<r_{0}, b_{0}<1 / 2$ depending only on $M, B$ and $n$ such that

$$
\alpha(\nabla u, \nu) \leq b_{0} \delta \text { in } B_{1 / 8}\left(\frac{3}{4} e_{n}\right) \times\left(-r_{0} \frac{\delta}{\mu}, r_{0} \frac{\delta}{\mu}\right) .
$$

for a unit vector $\nu$. (In other words, away from the boundary, $u$ is monotone for the cone $W\left(\pi / 2-b_{0} \delta, \nu\right)$.) 
Define

$$
\mathcal{N}=\left\{x: \alpha(x, y) \leq b_{0} \delta\right\},
$$

where $y$ is a unit vector on the touching line (if they touch) $\partial W\left(\theta / 2,-e_{n}\right) \cap$ $\partial W\left(\pi / 2-b_{0} \delta-\theta / 2, \nu\right)$.

Let us consider $p \in W\left(\theta / 2,-e_{n}\right)-\mathcal{N}$ and define $\sigma$ as

$$
\sigma=\pi / 2-\theta / 2-\alpha(p, \nu) \geq b \delta .
$$

For each $t \in\left(-r_{0} \frac{\delta}{\mu}, r_{0} \frac{\delta}{\mu}\right)$, let

$$
u_{1}(x, t)=u(x-p, t) \text { and } \epsilon=|p| \sin \theta / 2 .
$$

By definition of $\epsilon, p+\epsilon \xi \in W\left(\theta,-e_{n}\right)$ for any unit vector $\xi \in \mathbb{R}^{n}$ and thus

$$
v_{\epsilon} \leq u \text { in } B_{1}(0) \times(-r, r) .
$$

Moreover if $\bar{p}=p+(1+\sigma) h \epsilon$ and $h \leq \frac{1}{10}$, then $\alpha(\bar{p}, p) \leq \theta / 2+\frac{b_{0}}{3} \delta$ and thus

$$
\alpha(\bar{p}, \nu) \leq \alpha(\bar{p}, p)+\alpha(p, \nu) \leq \pi / 2-b_{0} \delta-\frac{1}{3} \sigma,
$$

that is, $\bar{p}$ is in the interior cone of monotonicity $W\left(\pi / 2-b_{0} \delta, \nu\right)$ away from the boundary of the cone by $\frac{1}{3} \sigma$. Hence a straighforward computation (see the proof of Lemma 4.2 in [K2]) yields

$$
u(x-\bar{p}, t) \leq u(x, t)-\operatorname{c\sigma \epsilon } u(x, t) \text { in } B_{1 / 8}\left(-\frac{3}{4} e_{n}\right) \times(-r, r) .
$$

Hence (9.2) and (9.3) is satisfied for our choice of $u_{1}$ and $u_{2}:=u$. Now we can apply Lemma 9.3 and proceed as in the proof of Lemma 4.2 in [K2] to conclude.

\section{Iteration}

Our goal in this section is to prove the following theorem.

Theorem 10.1. Let $P_{0} \in \Gamma_{0}$ and choose sufficiently small $r>0$ such that $t\left(P_{0}+r e_{n}\right) \leq t \leq t\left(P_{0}+2 r e_{n}\right)<t_{0}$. Then, for $x \in \Gamma_{t}(u) \cap B_{3 r}\left(P_{0}\right), \nabla u(x, t)$ exists as the nontangential limit from $\Omega_{t}(u)$ and satisfies

$$
|\nabla u(x, t)| \leq C\left|\nabla u_{0}\left(P_{0}-r e_{n}\right)\right|
$$

where $C$ is a constant depending only on $\bar{r}, \bar{R}, \bar{M}$ and $n$. 
Recall that the other direction of (10.1) (that is, the lower bound of $|\nabla u|)$ has been proved in Corollary 7.2.

Let us fix

$$
s_{0} \in\left[t\left(P_{0}+r e_{n}\right), t\left(P_{0}+2 r e_{n}\right)\right] \text { and } x_{0} \in \Gamma_{s_{0}}(u) \cap B_{3 r}\left(P_{0}\right) .
$$

For the rest of the section $C$ denotes various positive constants depending only on $\bar{r}, \bar{R}, \bar{M}$ and $n$. Change the coordinates and rescale so that $\left(x_{0}, s_{0}\right)=$ $(0,0)$ and $r=1 / 4$. By Corollary 2.2

$$
1 / C \leq\left|\nabla u\left(-e_{n},-1\right)\right|:=m_{0} \leq C .
$$

Hence Theorem 10.1 is proved if we can show that

$$
|\nabla u(0,0)| \leq C .
$$

First we prove that $\Gamma$ is differentiable in space.

Theorem 10.2. $\Gamma(u)$ is differentiable in space at $(0,0)$. In particular, there exist constants $l_{0}, c>0$ depending only on $\bar{r}, \bar{R}, \bar{M}$ and $n$ such that $\Gamma_{0}(u) \cap$ $B_{2^{-l}}(0)$ is a Lipschitz graph with Lipschitz constant less then $\frac{1}{l m_{0}}$ if $l \geq l_{0}$.

Proof. First, we construct a blow-up family $\left\{u_{l}\right\}$ of $u$ which satisfy the hypothesis of Lemma 9.3 as follows:

$$
u_{l+1}(x, t):=\alpha_{l} u\left(2^{-l} x, 4^{-l} \alpha_{l} t\right),
$$

where $\alpha_{0}=1$ and for $l \geq 1$

$$
\alpha_{l}= \begin{cases}2^{l} & \text { if } m_{0} \leq|\nabla u|\left(-2^{-l} e_{n}, 0\right) \leq 1 \\ \frac{2^{l}}{|\nabla u|\left(-2^{-l} e_{n}, 0\right)} & \text { otherwise }\end{cases}
$$

Note that $u_{l}(l=1,2 \ldots)$ is a viscosity solutions of (HS) in $B_{1}(0) \times(-1,1)$ with the property $m_{0} \leq\left|\nabla u_{l}\right|\left(-e_{n}, 0\right) \leq 1$. Hence by Corollary 7.2 , we obtain

$$
\left|\nabla u_{l}(x, t)\right| \geq C m_{0} \text { for }(x, t) \in\left(B_{1}(0) \cap \Omega_{t}\left(u_{l}\right)\right) \times(-1 / 2,1) .
$$

Due to Lemma 8.3,

$$
0 \leq \frac{\left(u_{l}\right)_{t+}}{\left|\nabla u_{l}\right|} \leq C\left|\nabla u_{l}\right| \leq C \text { in } B_{1 / 8}\left(-\frac{3}{4} e_{n}\right) \times(-1,1) .
$$


Hence by Lemma 9.3, for each $l>0 u$ is monotone for the cone $W\left(\nu_{l+1}, \theta_{l}\right)$ in in

$$
B_{2^{-l}}(0) \times\left[-4^{-l} \frac{\alpha_{l} \delta_{l}}{C}, 4^{-l} \frac{\alpha_{l} \delta_{l}}{C}\right],
$$

where $\delta_{l}=\pi / 2-\theta_{l}$ satisfies

$$
\delta_{l+1}=\delta_{l}-c \delta_{l}^{2} / C .
$$

By (10.3) we obtain $\delta_{l} \approx \frac{C}{l}$, which yields the differentiability of $\Gamma(u)$ in space at $(0,0)$.

From above argument,

$$
\Gamma_{s_{0}}(u) \cap B_{2 r}\left(P_{0}\right)=\left\{x_{n}=f\left(x^{\prime}\right)\right\} \cap B_{2 r}\left(P_{0}\right)
$$

is differentiable with the estimate

$$
\left|\nabla f\left(x^{\prime}\right)-\nabla f\left(y^{\prime}\right)\right| \leq\left(-\log \left|x^{\prime}-y^{\prime}\right|\right)^{-1} .
$$

The continuity mode of $\nabla \varphi$ obtained above is not strong enough to finish the proof of Theorem 10.1. More precisely, if we knew that $\delta_{l}$ obtained from (10.3) satisfied

$$
\delta_{l} \approx l^{-m} \text { for some } m>1
$$

then Theorem 2.4 of Widman [W] would show that $\nabla u$ exists up to the free boundary $\Gamma(u)$ as the nontangential limit from $\Omega(u)$ and $|\nabla u| \leq C u\left(-e_{n}, 0\right)$ for some $C$ depending on $p$. Since we only have $\delta_{l} \approx l^{-1}$ we need to iterate a second time with paying close attention to the range of $u_{t}$. First we state a refined version of Lemma 8.3.

Lemma 10.3. Let $u$ be given as in Theorem 2.1. Suppose that $\Gamma_{t_{1}}(u), t_{1}>$ 0 , is differentiable at $x_{1}$ with the inward unit normal vector $\nu$. Furthermore suppose that $\nu \cdot \nabla u\left(\cdot, t_{1}\right)$ has the nontangential limit $a_{0}$ from $\Omega_{t_{1}}(u)$ at $x_{1}$. Then $\Gamma \cap\left\{t \geq t_{1}\right\}$ is differentiable at $\left(x_{1}, t_{1}\right)$ and

$$
u(x, t)=a_{0}\left[\left(x-x_{1}\right) \cdot \nu-a_{0}\left(t-t_{1}\right)\right]_{+}+o\left(\left|x-x_{1}\right|+\left|t-t_{1}\right|\right) \text { for } t \geq t_{1} .
$$

Proof. We change the coordinate to set $a_{0}=1,\left(x_{1}, t_{1}\right)=(0,0), \nu=-e_{n}$ and $x_{n}=x \cdot e_{n}$. Consider the blow-up sequence

$$
u^{n}(x, t)=3^{n} u\left(3^{-n} x, 3^{-n} t\right) \text { in } B_{2^{-n}}(0) \times\left(-2^{n}, 2^{n}\right), n=1,2, \ldots
$$


We consider

$$
\bar{u}(x, t):=\limsup _{(y, s) \rightarrow(x, t)} \limsup _{n \rightarrow \infty} u^{n}(y, s)
$$

and

$$
\underline{u}(x, t):=\liminf _{(y, s) \rightarrow(x, t)} \liminf _{n \rightarrow \infty} u^{n}(y, s) .
$$

By the nondegeneracy of $u$ and the stability property of viscosity solutions, $\bar{u}$ and $\underline{u}$ are respectively a subsolution and a supersolution of (HS). Due to Theorem 5.7 and Theorem $6.1, \bar{u}$ and $\underline{u}$ is monotone along directions in $W\left(\pi / 2, e_{n}\right)$. Hence $\bar{u}(x, t)=\bar{u}\left(x_{n}, t\right)$ and $\underline{u}(x, t)=\underline{u}\left(x_{n}, t\right)$. In particular $\bar{u}$ and $\underline{u}$ has its free boundary as half-space at each time, respectively

$$
\left\{x_{n}=\alpha(t)\right\} \text { and }\left\{x_{n}=\beta(t)\right\}, \quad \alpha(0)=\beta(0)=0 .
$$

By a barrier argument it follows that $\alpha(t)$ and $\beta(t)$ are continuous. From Lemma 1.11, Theorem 2.1 and Lemma 8.3, it follows that $\left\{u^{n}\right\}$ is uniformly Lipschitz in space and time on

$$
S:=\left\{x:-2 \leq x_{n} \leq-1 / 2\right\} \times(-c, c)
$$

for sufficiently small $c$. In particular,

$$
\bar{u}(x, t)=\underline{u}(x, t)=a(t) \text { on }\left\{x: x_{n}=-1\right\} \times(-c, c)
$$

where $a(t)$ is Lipschitz continuous in time with $a(0)=1$. It follows from the maximum principle of harmonic functions that $\bar{u}\left(x_{n}, 0\right) \leq \underline{u}\left(x_{n}, 0\right)$. Since $\bar{u} \geq \underline{u}$ by definition, it follows that $\bar{u}\left(x_{n}, 0\right)=\underline{u}\left(x_{n}, 0\right)$.

Next observe that

$$
w_{1}(x, t):=\frac{a(t)}{1+\alpha(t)}\left(-x_{n}+\alpha(t)\right)_{+} \text {and } w_{2}(x, t):=\frac{a(t)}{1+\beta(t)}\left(-x_{n}+\beta(t)\right)_{+}
$$

satisfies $\bar{u} \leq w_{1}$ and $w_{2} \leq \underline{u}$ in $D:=\left\{x: x_{n} \geq-1\right\} \times[0, c)$ by applying the maximum principle of harmonic functions at each time. Since $\bar{u}(\underline{u})$ and $w_{1}\left(w_{2}\right)$ has the same free boundary, it follows that $w_{1}$ and $w_{2}$ are respectively viscosity sub- and supersolutions of (HS) in $D$ with $w_{1} \leq w_{2}$ on the parabolic boundary of the domain. Therefore by Theorem 1.8, $w_{1} \leq w_{2}$ for $t>0$ and thus $\bar{u}=w_{1}=w_{2}=\underline{u}$ for $t>0$ in $D$.

So far we showed that $\left\{u^{n}\right\}$ locally uniformly converges to a viscosity solution of (HS)

$$
u^{0}(x, t)=\frac{a(t)}{1+\alpha(t)}\left(-x_{n}+\alpha(t)\right)_{+}
$$


in $D$, where $a(t)$ is Lipschitz continuous, $a(0)=1$ and $\alpha(0)=0$. Recall that $u^{0}$ is Lipschitz continuous in time in $\left\{(x, t):-2 \leq x_{n} \leq-1 / 2\right\}$ for small $t$. Thus from a simple barrier argument it follows that $\alpha(t)$ is also Lipschitz continuous for small $t$, and $\left(u^{0}\right)^{n}$ locally uniformly converges to

$$
u^{00}(x, t)=\left(-x_{n}+b^{0}(t)\right)_{+} \text {in } \mathbb{R}^{n} \times \mathbb{R}
$$

where $b^{0}(t)$ is continuous with $b^{0}(0)=0$. Lastly, by comparing $u^{00}$ with barriers of type $(a(0) \pm \epsilon)\left(-x_{n}+(a(0) \pm \epsilon) t\right)_{+}$, it follows that $b^{0}(t) \equiv t$ and we can conclude.

Let $k(x, y ; t)$ be the Poisson kernel of $\Omega_{t}$ with pole $x \in \Omega_{t}$ evaluated at $y \in \Gamma_{t}$. That is, if $g$ is a harmonic function in $D$ with boundary data $f$ on $\partial D$, then

$$
g(x)=\int_{\partial D} k(x, y ; t) f(y) d \sigma_{t}(y),
$$

where $d \sigma_{t}(y)$ is the surface measure of $\partial D$.

\section{Lemma 10.4.}

$$
u_{t+}(x, t)=\int_{\Gamma_{t}} k(x, y ; t)|\nabla u|^{2}(y, t) d \sigma_{t}(y) .
$$

where $\nabla u(y, t)$ is defined almost everywhere on $\Gamma_{t}$ as the nontangential limit from $\Omega_{t}$.

Proof. Take any sequence $\epsilon_{m}>0$ which converges to zero as $m \rightarrow \infty$ and let

$$
f_{m}(x, t)=\frac{u\left(x, t+\epsilon_{m}\right)-u(x, t)}{\epsilon_{m}} \text { for } x \in \Omega_{t} .
$$

The same reasoning as in the proof of Lemma 8.3 gives for $y \in \Gamma_{t}$,

$$
f_{m}(y, t)=\frac{u\left(y, t+\epsilon_{m}\right)}{\epsilon_{m}} \leq C\left|\nabla u\left(y, t+\epsilon_{m}\right)\right|^{2} \stackrel{g}{\approx}\left|\nabla u\left(y^{\prime}, t\right)\right|^{2} \leq\left|(\nabla u)^{\star}(y, t)\right|^{2}
$$

where $y^{\prime}$ is a point in $\Omega_{t}$ such that

$$
\operatorname{dist}\left(y^{\prime}, \Gamma_{t}\right) \approx\left|y-y^{\prime}\right|=\operatorname{dist}\left(y, \Gamma_{t+\epsilon_{m}}\right)
$$

and $(\nabla u)^{\star}$ is the nontangential maximal function of $\nabla u$. 
The right side of above inequality is $L^{1}\left(d \omega^{x}(y)\right)$ and Lemma 10.3 implies that $f_{n}(\cdot, t)$ converges to $|\nabla u|^{2}(\cdot, t)$ (given as the nontangential limit from $\Omega_{t}$ ) almost everywhere on $\Gamma_{t}$. Hence by dominated convergence theorem,

$$
\lim _{n \rightarrow \infty} \int_{\Gamma_{t}} f_{n}(y, t) d \omega^{x}(y)=\int_{\Gamma_{t}}|\nabla u|^{2}(y, t) d \omega^{x}(y) .
$$

Proposition 10.5. Suppose $0 \in \Gamma_{t}, t>0$ and $u(\cdot, t)$ satisfies $\alpha\left(\nabla u,-e_{n}\right) \leq$ $\frac{1}{l}, l>1$ in

$$
D_{l}:=\Omega_{t} \cap\left\{\left(x^{\prime}, x_{n}\right) \in \mathbb{R}^{n}:\left|x^{\prime}\right| \leq 2^{-l} \sqrt{l},\left|x_{n}\right| \leq 2^{-l}\right\} .
$$

Let $\beta=|\nabla u|\left(-2^{-l} e_{n}, t\right)$, then there is a constant $0<p<1$ depending only on $\bar{r}, \bar{R}, \bar{M}$ and $n$ such that

$$
u_{t}(x, t)=\beta^{2}\left(1+O\left(l^{-p}\right)\right) \text { for } x \in B_{2^{-l} / 8}\left(-2^{-l} e_{n}\right) .
$$

Proof. By Lemma 10.4,

$$
u_{t+}(x, t)=\int_{\Gamma_{t}} k(x, y ; t)|\nabla u|^{2}(y, t) d \sigma_{t}(y) .
$$

Thus our proposition follows from the following two lemmas in which we estimate the integrand in (10.4) in the domain $D_{l} \cap \Gamma_{t}$ and $\Gamma_{t}-D_{l}$ respectively.

\section{Lemma 10.6.}

$$
\int_{\Gamma_{t}-D_{l}} k(x, y ; t)|\nabla u(y, t)|^{2} d \sigma_{t}(y) \leq C \frac{|\nabla u(x, t)|^{2}}{l^{p}}
$$

where $C>0$ and $0<p<1$ are constants depending only on $\bar{r}, \bar{R}, \bar{M}$ and $n$.

Proof. Define $B_{i}=B_{2^{i} 2^{-l} \sqrt{l}}(x), i=0,1,2, .$. , and let $z_{i}$ be a point in the middle of $\Omega_{t} \cap\left(B_{i}-B_{i-1}\right)$. Then for any $y \in\left(B_{i}-B_{i-1}\right) \cap \Gamma_{t}$,

$$
k(x, y ; t) \approx \omega\left(x, \partial B_{i}, B_{i} \cap \Omega_{t}\right) k\left(z_{i}, y ; t\right) .
$$

Hence for $x_{0} \in \Omega_{t}(u)$ a unit distance away from $\Gamma_{t}(u)$, 


$$
\begin{aligned}
& \int_{\Gamma_{t}-D_{l}} k(x, y ; t)|\nabla u(y, t)|^{2} d \sigma_{t}(y) \\
\approx & \sum_{i} \int_{\Gamma_{t} \cap\left(B_{i}-B_{i-1}\right)} \omega\left(x, \partial B_{i}, B_{i} \cap \Omega_{t}\right) k\left(z_{i}, y ; t\right)|\nabla u(y, t)|^{2} d \sigma_{t}(y) \\
\leq & \sum_{i} \omega\left(x, \partial B_{i}, B_{i} \cap \Omega_{t}\right)\left|\nabla u\left(z_{i}, t\right)\right|^{2} \\
\approx & \sum_{i} \omega\left(x, \partial B_{i}, B_{i} \cap \Omega_{t}\right)\left(\frac{u\left(z_{i}, t\right)}{2^{i-l} \sqrt{l}}\right)^{2} \\
\approx & \sum_{i} u(x, t) \frac{u\left(z_{i}, t\right)}{\left(2^{i-l} \sqrt{l}\right)^{2}} .
\end{aligned}
$$

where the first inequality is due to the Reversed Hölder Inequality (see Lemma 8.1 for $n=2$ and [D2] for $n \geq 3)$. Since $u\left(z_{i}, t\right) \leq C u(x, t)\left(2^{i} \sqrt{l}\right)^{1+a}$ for some $a \in(0,1)$,

$$
\begin{aligned}
\sum_{i} u(x, t) \frac{u\left(z_{i}, t\right)}{\left(2^{i-l} \sqrt{l}\right)^{2}} & \leq \frac{C}{l^{(1-a) / 2}} u^{2}(x, t) \sum_{i} 2^{2 l+i(1+a)-2 i} \\
& \leq \frac{C}{l^{(1-a) / 2}} 2^{2 l} u^{2}(x, t) \leq \frac{C}{l^{(1-a) / 2}}|\nabla u(x, t)|^{2} .
\end{aligned}
$$

Lemma 10.7. For $x \in B_{2^{-l} / 8}\left(2^{-l} e_{n}\right)$,

$$
\int_{D_{l} \cap \Gamma_{t}} k(x, y ; t)|\nabla u|^{2}(y, t) d \sigma_{t}(y)=\beta^{2}\left(1+O\left(l^{-p}\right)\right)
$$

for a constant $0<p<1$ depending only on $\bar{r}, \bar{R}, \bar{M}$ and $n$.

Proof. We will divide $D_{l}$ into little boxes $\left\{B_{i}\right\}\left(1 \leq i \leq l^{n-1}\right)$ with bottom $F_{i}:=B_{i} \cap\left\{x_{n}=-2^{-l}\right\}$ being a $n-1$ dimensional square with a dimension of $2^{-l} / \sqrt{l}$. Let $I_{i}:=B_{i} \cap \Gamma_{t}$ be the corresponding partition of the free boundary. Also let

$$
S_{i}=\partial\left(B_{i} \cap \Omega_{t}\right)-F_{i} \cup I_{i} .
$$

Define $u_{n}(x, t):=-e_{n} \cdot \nabla u(x, t)$. For each $i$, let $\beta_{i}=u_{n}\left(\left(x_{i}^{\prime},-2^{-l}\right), t\right)$ where $\left(x_{i}^{\prime},-2^{-l}\right)$ is the middle point of $F_{i}$. We first show that

$$
\left|I_{i}\right|^{-1} \int_{I_{i}} u_{n} d \sigma_{t}=\beta_{i}\left(1+O\left(l^{-1 / 2}\right)\right)
$$


To prove (10.5), we apply Green's theorem to obtain

$$
\begin{aligned}
0=\int_{B_{i} \cap \Omega_{t}} \Delta u & =\oint_{\partial\left(B_{i} \cap \Omega_{t}\right)} \nabla u \cdot \nu d \sigma_{t} \\
& =\int_{F_{i}} u_{n} d \sigma_{t}-\left(1+O\left(l^{-1}\right)\right) \int_{I_{i}} u_{n} d \sigma_{t}+\int_{S_{i}} \nabla u \cdot \nu d \sigma_{t}
\end{aligned}
$$

Since $\alpha\left(\nabla u,-e_{n}\right) \leq \frac{1}{l}$ and $\beta_{i} \approx \frac{u\left(\left(x_{i}^{\prime},-2^{-l}\right), t\right)}{2^{-l}}$,

$$
\begin{aligned}
\int_{S_{i}} \nabla u \cdot \nu d \sigma_{t} & \leq \frac{1}{l} \cdot \int_{\partial F_{i}} u d \mathcal{H}^{n-2} \\
& \leq \frac{1}{l} \cdot \mathcal{H}^{n-2}\left(\partial F_{i}\right) \cdot \sup _{F_{i}} u \leq \frac{C}{\sqrt{l}} \cdot \sigma_{t}\left(F_{i}\right) \cdot \beta_{i},
\end{aligned}
$$

where $\mathcal{H}^{n-2}$ denotes the $(n-2)$-dimensional Hausdorff measure. Hence we obtain

$$
\int_{I_{i}} u_{n} d \sigma_{t} \leq\left(1+O\left(l^{-1}\right)\right) \int_{F_{i}} u_{n} d \sigma_{t}+\frac{C}{\sqrt{l}} \cdot \sigma_{t}\left(F_{i}\right) \cdot \beta_{i}
$$

and this implies (10.5).

Next observe that by interior estimates of harmonic functions and by our hypothesis $\alpha\left(\nabla u,-e_{n}\right) \leq l^{-1}$,

$$
\left|u_{i j}\right| \leq C\left|u_{i}\right| \leq C l^{-1}\left|u_{n}\right| \text { on }\left\{x_{n}=-2^{-l}\right\} \cap D_{l}, \text { for } 1 \leq i \leq n-1
$$

where $C$ depends only on $\bar{r}, \bar{R}, \bar{M}$ and $n$. Since $u$ is harmonic in $\Omega(u)$, it follows that

$$
\left|u_{n n}\right| \leq C(n-1)\left|u_{n}\right| / l \text {. }
$$

As a consequence it follows that

$$
\beta_{i}=\beta\left(1+O\left(l^{-1 / 2}\right)\right) .
$$

Also observe that due to Corollary 3.3 in [JK1], for any $\epsilon>0$ there exist $\gamma>0$ such that if $d\left(x, \Gamma_{t}\right)=1$, and $I \subset B_{1}(0) \cap \Gamma_{t}$ satisfies $|I| \leq \gamma$, then

$$
|I|^{-1} \int_{I} k^{3}(x, y ; t) d \sigma_{t}(y) \leq(1+\epsilon)\left(|I|^{-1} \int k(x, y ; t) d \sigma_{t}(y)\right)^{3} .
$$

Let $x_{0}$ denote a fixed point in the middle of $\Omega_{0}$ and let

$$
K(x, y ; t):=k(x, y ; t) / k\left(x_{0}, y ; t\right) .
$$


Following the proofs in [JK1] one can check that $\delta=\gamma=\epsilon^{p_{1}}$ for some dimensional constant $p_{1}$. Hence we obtain

$$
\begin{aligned}
& \int_{D_{l} \cap \Gamma_{t}} k(x, y ; t)|\nabla u|^{2}(y, t) d \sigma_{t}(y) \\
= & \sum_{i}\left(1+O\left(l^{-1}\right)\right) \int_{I_{i}} K(x, y ; t) k\left(x_{0}, y ; t\right) u_{n}^{2}(y, t) d \sigma_{t}(y) \\
= & C_{0}\left(1+O\left(l^{-1}\right)\right) \sum_{i} \int_{I_{i}} K(x, y ; t) u_{n}^{3}(y, t) d \sigma_{t}(y) \\
= & C_{0}\left(1+O\left(l^{-p_{1}}\right)\right) \sum_{i} a_{i} \int_{I_{i}} u_{n}^{3}(y, t) d \sigma_{t}(y) \\
= & C_{0}\left(1+O\left(l^{-p_{2}}\right)\right) \sum_{i} a_{i}\left|I_{i}\right|\left[\left|I_{i}\right|^{-1} \int_{I_{i}} u_{n}(y, t) d \sigma_{t}(y)\right]^{3} \\
= & C_{0}\left(1+O\left(l^{-p_{3}}\right)\right) \sum_{i} a_{i}\left|I_{i}\right| \beta_{i}^{3} \\
= & C_{0}\left(1+O\left(l^{-p_{4}}\right)\right) \sum_{i} \int_{I_{i}} K(x, y ; t) \beta_{i}^{3} d \sigma_{t}(y) \\
= & \left(1+O\left(l^{-p_{4}}\right)\right) \beta^{2} \sum_{i} \int_{I_{i}} K(x, y ; t) k\left(x_{0}, y ; t\right) d \sigma_{t}(y) \\
= & \left(1+O\left(l^{-p_{5}}\right)\right) \beta^{2}
\end{aligned}
$$

where $a_{i}=\left|I_{i}\right|^{-1} \int_{I_{i}} K(x, y ; t) d \sigma_{t}(y)$. Here the second equality is due to

$$
k\left(x_{0}, y ; t\right)=C_{0}\left(1+O\left(l^{-1}\right)\right) u_{n} \text { a.e. for } C_{0}=C\left(x_{0}\right),
$$

the third and the sixth are due to the $C^{\alpha}$-regularity of $K$ in $y$-variable (see [JK2]), the fourth is due to (10.7), the fifth is due to (10.5), the seventh is due to $(10.6),(10.8)$ and the $C^{\alpha}$-regularity of $K$ in $y$-variable. To prove the eighth equality we use the inequality

$$
\int_{\Gamma_{t}-D_{l}} k(x, y ; t) d \sigma_{t}(y) \leq C \frac{1}{p_{6}},
$$

the proof of which is parallel to that of Lemma 10.6.

Proof of Theorem 10.1 From Proposition 10.5 and the first iteration

$$
\frac{u_{t}}{|\nabla u|}(x, t)=|\nabla u|\left(\left(2^{l} \sqrt{l}\right)^{-1} \nu_{n}, t\right)\left(1+O\left(l^{-p}\right)\right), 0<p<1
$$


in the region

$$
B_{\frac{1}{8}\left(2^{l} \sqrt{l}\right)^{-1}}\left(\left(2^{l} \sqrt{l}\right)^{-1} \nu_{n}\right) \times\left[-\frac{\alpha_{l}}{4^{l} l}, \frac{\alpha_{l}}{4^{l} l}\right]
$$

where $\alpha_{l}$ is defined as in (10.2) and $\nu_{n}$ is obtained from the first iteration.

Now based on (10.9), we iterate Lemma 9.3 the second time with the sequence of functions

$$
\tilde{u}^{l+1}(x, t)=\beta_{l} u\left(\frac{x}{2^{l} \sqrt{l}}, \frac{\beta_{l} t}{4^{l} l}\right)
$$

in $B_{1}(0) \times\left[-\alpha_{l} / \beta_{l}, \alpha_{l} / \beta_{l}\right]$ where

$$
\beta_{l}= \begin{cases}2^{l} \sqrt{l} & \text { if } m_{0} \leq|\nabla u|\left(-\left(2^{l} \sqrt{l}\right)^{-1} e_{n}, 0\right) \leq 1 \\ \frac{2^{l} \sqrt{l}}{|\nabla u|\left(-\left(2^{l} \sqrt{l}\right)^{-1} e_{n}, 0\right)} & \text { otherwise }\end{cases}
$$

Namely, $\left\{\tilde{u}^{l}\right\}$ is defined similarly as $\left\{u^{l}\right\}$ in (10.2), but replacing the scaling factor $2^{l}$ by $2^{l} \sqrt{l}$. Now to see if each $\tilde{u}^{l}$ satisfies the hypotheses of Lemma 9.1 with $r=\alpha_{l} / \beta_{l}$ and $\mu=\mu_{l}=l^{-p^{\prime}}, p^{\prime}=\min (p, 1 / 3)$, we only have to check if $\alpha_{l} / \beta_{l} \geq \delta_{l} / \mu_{l}$. Note that due to Corollary 7.2

$$
\begin{aligned}
\alpha_{l} / \beta_{l} & =C l^{-1 / 2} \frac{|\nabla u|\left(-\left(2^{l} \sqrt{l}\right)^{-1} \nu_{n}, 0\right)}{|\nabla u|\left(-2^{-l} \nu_{n}, 0\right)} \\
& \geq C \frac{m_{0}}{\sqrt{l}} \\
& \geq C l^{-1 / 2} \geq \delta_{l} / \mu_{l}
\end{aligned}
$$

for sufficiently large $l$, since $\delta_{l} \leq l^{-1}$ from our first iteration. Hence the iteration is valid and we obtain

$$
\delta_{l+1}=\delta_{l}-\delta_{l}^{2} / \mu_{l} .
$$

From (10.10) it follows that $\delta_{l} \approx l^{-m}, 1<m<2$. Now Theorem 10.1 follows from our estimates and Theorem 2.4 of $[\mathrm{W}]$.

Corollary 10.8. Let $u$ be as in Theorem 2.1. Then there exists $t_{0}$ depending only on $\bar{r}, \bar{R}, \bar{M}$ and $n$ such that

(a) $\Gamma(u)$ is differentiable in space and time for $0<t \leq t_{0}$. 
(b) $u_{t}, \nabla u$ exist in $\bar{\Omega} \times\left(0, t_{0}\right]$.

(c) $u$ satisfies the free boundary condition $u_{t}=|\nabla u|^{2}$ in the classical sense.

(In other words $u$ is the classical solution of (HS) for $0<t \leq t_{0}$.)

Proof. Due to Theorem 10.1 and Lemma 8.3, $|\nabla u|$ and $u_{t}$ are bounded from above. Now (a) and (b) are obtained from the iteration process given above with $\delta_{l}, \mu_{l} \sim l^{-m} \rightarrow 0$ as $l \rightarrow \infty$. (c) follows from Lemma 10.3.

Corollary 10.9. Let $u$ be given as in Theorem 2.1 with $P_{0}=0$. There are positive constants $r_{1}, C_{1}, C_{2}$ only depending on $\bar{r}, \bar{R}, \bar{M}$ and $n$ such that, for $x \in B_{r}\left(r e_{n}\right) \cap \Omega_{t\left(r e_{n}\right)}$ with $0<r<r_{1}$,

$$
C_{1}|\nabla u|^{2}\left(-r e_{n}, 0\right) \leq u_{t}(x, t) \leq C_{2}|\nabla u|^{2}\left(-r e_{n}, 0\right)
$$

and

$$
C_{1}|\nabla u|\left(-r e_{n}, 0\right) \leq|\nabla u|(x, t) \leq C_{2}|\nabla u|\left(-r e_{n}, 0\right)
$$

Proof. (10.11) follows from Theorem 7.1, Lemma 8.3 and Theorem 10.1. (10.12) is due to Corollary 7.2, and Theorem 10.1.

\section{References}

[ACS] I. Athanasopoulos, L. Caffarelli and S. Salsa, Regularity of the free boundary in parabolic phase-transition problems, Acta Math., 176(1996), 245-282.

[C1] L. Caffarelli, A Harnack inequality approach to the regularity of free boundaries, Part I: Lipschitz free boundaries are $C^{1, \alpha}$, Rev. Mat. Iberoamericana3 (1987), no. 2, 139-162

[C2] L. Caffarelli, A Harnack inequality approach to the regularity of free boundaries, Part II: Flat free boundaries are Lipschitz,Comm. Pure Appl. Math., 42(1989), 55-78.

[D] B. Dahlberg, Harmonic functions in Lipschitz domains, Harmonic analysis in Euclidean spaces, Part 1, pp. 313-322, Proc. Sympos. Pure Math., XXXV, Part, Amer. Math. Soc., Providence, R.I., 1979 
[D2] B. Dahlberg, On the Poisson integral for Lipschitz and $C^{1}$ domains, Studia Math, 66 (1979), 13-24.

[EJ] C. M. Elliot and V. Janovsky, A variational inequality approach to Hele-Shaw flow with a moving boundary, Proc. Roy. Soc. Edinburgh. Sect. A 88 (1981), no.1-2, 93-107.

[ES] J. Escher, G. Simonett, Classical solutions of multidimensional HeleShaw models, SIAM J. Math. Anal 28 (1997), no.5, pp. 1028-1047.

[HMW] R. Hunt, B. Muckenhoupt and R. Wheeden, Weighted norm inequalities for the conjugate function and Hilbert transform, Trans. AMS 176 (1973), 227-251.

[HS] H. Helson and G. Szegö, A problem in prediction theory, Ann. Mat. Pura. Appl. (4) 51 (1960) 107-138.

[JK1] D. S. Jerison and C. E. Kenig, The logarithm of the Poisson kernel of a $C^{1}$ domain has vanishing mean oscillation, Trans. Amer. Math. Soc. 273 (1982), 781-794.

[JK2] D. S. Jerison and C. E. Kenig, Boundary behavior of Harmonic functions in Non-tangentially Accessible Domains, Advan. in Math. 46 (1982), 80-147.

[JK3] D. S. Jerison and C. E. Kenig, Boundary value problems on Lipschitz domains, Studies in Partial Di erential Equations, ed. W. Littman, Math. Assoc. Amer. (1982), 1-68.

[JeKi] D. S. Jerison and I. Kim, The one-phase Hele-Shaw problem with singularities, preprint.

[K1] I. Kim, Uniqueness and Existence result of Hele-Shaw and Stefan problem, Arch. Rat. Mech. Anal, 168 (2003), 299-328.

[K2] I. Kim, Regularity of free boundary in one phase Hele-Shaw problem, submitted

[W] K. -O. Widman., Inequalities for the Green function and boundary continuity of the gradient of solutions of elliptic differential equations, Math. Scand., 21 (1967), 17-37. 